\title{
Rigorous numerics in Floquet theory: computing stable and unstable bundles of periodic orbits
}

\author{
Roberto Castelli * Jean-Philippe Lessard ${ }^{\dagger}$
}

\begin{abstract}
In this paper, a rigorous method to compute Floquet normal forms of fundamental matrix solutions of non-autonomous linear differential equations with periodic coefficients is introduced. The Floquet normal form of a fundamental matrix solution $\Phi(t)$ is a canonical decomposition of the form $\Phi(t)=Q(t) e^{R t}$, where $Q(t)$ is a real periodic matrix and $R$ is a constant matrix. To compute rigorously the Floquet normal form, the idea is to use the regularity of $Q(t)$ and to solve simultaneously for $R$ and $Q(t)$ with the contraction mapping theorem in a Banach space of rapidly decaying coefficients. The explicit knowledge of $R$ and $Q$ can then be used to construct, in a rigorous computer-assisted way, stable and unstable bundles of periodic orbits of vector fields. The new proposed method does not require rigorous numerical integration of the ODE.
\end{abstract}

\section{Keywords}

Rigorous numerics - Floquet theory - Fundamental matrix solutions ·

Contraction mapping theorem · Periodic orbits · Tangent bundles

\section{Mathematics Subject Classification (2000)}

37B55 - 37M99 - 37C27 - 65G99 - 34D05

\section{Introduction}

In his seminal work [1] of 1883, Gaston Floquet studied linear non-autonomous differential equations of the form

$$
\dot{y}=A(t) y,
$$

where $A(t)$ is a $\tau$-periodic continuous matrix function of $t$. More precisely, Floquet introduces a canonical decomposition of the fundamental matrix solutions of (1).

Theorem 1.1. [Floquet Theorem] Let $A(t)$ be a $\tau$-periodic continuous matrix function and denote by $\Phi(t)$ a fundamental matrix solution of (1). Then $\Phi(t+\tau)$ is also a fundamental matrix solution, $\Phi(t+\tau)=\Phi(t) \Phi^{-1}(0) \Phi(\tau)$, and there exist a real constant matrix $R$ and a real nonsingular, continuously differentiable, $2 \tau$-periodic matrix function $Q(t)$ such that

$$
\Phi(t)=Q(t) e^{R t} .
$$

\footnotetext{
*Basque Center for Applied Mathematics (BCAM), Alameda de Mazarredo 14, 48009, Bilbao, Basque Country, Spain. Email: rcastelli@bcamath.org.

†Université Laval, Département de Mathématiques et de Statistique, 1045 avenue de la Médecine, Québec, Québec, G1V 0A6, Canada and Basque Center for Applied Mathematics (BCAM), Alameda de Mazarredo 14, 48009, Bilbao, Basque Country, Spain. Email: jean-philippe.lessard@mat.ulaval.ca. This work was partially supported by Grant MTM2011-24766 of the MICINN, Spain.
} 
The proof can be found for instance in [2]. The decomposition (2) is called a Floquet normal form for the fundamental matrix solution $\Phi(t)$. The real time-dependent change of coordinates $z=Q^{-1}(t) y$ transforms system (1) into a linear constant coefficients system of the form $\dot{z}=R z$. A stability theorem demonstrates that the linear stability of the zero solution of (1) can be determined by the eigenvalues of the so-called monodromy matrix $\Phi(\tau)$. While there has been several methods to compute (even rigorously) fundamental matrix solutions of (1), we are not aware of any rigorous method to compute Floquet normal forms as introduced in Theorem 1.1. This is one of the goal of the present work.

The motivation for developing such computational method comes from the study of dynamical systems, where (1) arises naturally when studying linear stability of periodic solutions of vector fields $\dot{y}=g(y)$, where $g: \mathbb{R}^{n} \rightarrow \mathbb{R}^{n}$ is a smooth map. Assume that $\Gamma$ is a $\tau$-periodic orbit of $\dot{y}=g(y)$ parameterized by $\gamma(t) \in \mathbb{R}^{n}(t \in[0, \tau])$, and define the $\tau$-periodic matrix function $A(t)=\nabla g(\gamma(t))$, where $\nabla g$ is the Jacobian matrix. Consider $\Phi(t)$ the principal fundamental matrix solution of $\dot{y}=A(t) y=\nabla g(\gamma(t)) y$, that is the unique fundamental matrix solution so that $\Phi(0)=I_{n}$, and assume that a Floquet normal form $\Phi(t)=Q(t) e^{R t}$ is known. The information from the Floquet normal form can be used to compute important dynamical properties of $\Gamma$ : the linear stability of the periodic orbit $\Gamma$ can be determined by the eigenvalues of $R$ while the stable and unstable tangent bundles of $\Gamma$ can be retrieved from the action of $Q(t)$ (with $t \in[0, \tau]$ ) on the eigenvectors of $R$ (e.g. see Theorem 3.3). Also, using the parameterization method introduced in [3], the higher order terms of a parameterization of an orientable invariant manifold of a periodic orbit can be computed efficiently from the action of $Q(t)$ on some given constant vectors. Therefore one motivation for developing computer-assisted proofs for Floquet normal forms is to compute rigorously high order parameterizations of invariant manifolds of periodic orbits.

Before proceeding further, let us mention that rigorous methods like the $C^{1}$-Lohner algorithm [4] can be used to study the linear stability of periodic orbits. For instance, a method combining multiple shooting, the interval Krawczyk method and the use of the $C^{1}$ Lohner algorithm to integrate the flow was introduced in [5] to obtain rigorous estimates for the monodromy matrix. An important difference is that our method does not rely on a rigorous time integration of the flow. Another important motivation for developing such method is that the computation of invariant bundles of periodic orbits is one of the key ingredient and one of the main difficulty in applying the parameterization method to compute invariant manifold of periodic orbits of a concrete equation (e.g. see $[6,7,8]$ ). In fact, once the invariant bundles are explicitly known, the computation of higher order terms is rather straightforward. Hence, we believe that developing a general method to compute Floquet normal form is important.

Let us now introduce the ideas behind the rigorous method. The first step is to substitute the Floquet normal form $\Phi(t)=Q(t) e^{R t}$ in the differential equation (1). From this, it follows that $(R, Q(t))$ is a solution of the differential equation with periodic coefficients $\dot{Q}=A(t) Q-Q R$. On the converse, if a real constant matrix $R$ and a $2 \tau$-periodic matrix function $Q(t)$ solve

$$
\left\{\begin{array}{l}
\dot{Q}=A(t) Q-Q R \\
Q(0)=I_{n}
\end{array}\right.
$$

then the matrix function $\Phi(t):=Q(t) e^{R t}$ is the principal fundamental solution of (1). Therefore, the problem of computing fundamental matrix solutions in the form $\Phi(t)=$ $Q(t) e^{R t}$ reduces to find $(R, Q(t))$ satisfying (3). The next step is to introduce a nonlinear operator $f$ (see Section 2.1 for details) whose zeros are in one-to-one correspondence with the solutions of (3). Letting $x=\left(R, \mathcal{Q}_{0}, \mathcal{Q}_{1}, \mathcal{Q}_{2}, \ldots\right)$, where the $\mathcal{Q}_{k}$ 's are the Fourier coefficients of $Q(t)$, the problem of computing Floquet normal forms $\Phi(t)=Q(t) e^{R t}$ is then equivalent 
to find $x$ such that $f(x)=0$. By the a priori knowledge of the smoothness of $Q(t)$, the Fourier coefficients $\mathcal{Q}_{k}$ 's decay fast, meaning that the solutions of $f(x)=0$ live in a suitable Banach space $\Omega^{s}$ of rapidly decaying coefficients. To prove existence, in a constructive way, of solutions of the infinite dimensional nonlinear operator equation $f(x)=0$ in $\Omega^{s}$, we use the so-called notion of rigorous numerics. To be more precise, rigorous numerics aims at constructing algorithms that provide an approximate solution to a problem together with precise bounds within which the exact solution is guaranteed to exist in the mathematically rigorous sense. It is worth mentioning that by now, the use of rigorous numerical methods is a standard approach to study differential equations and dynamical systems (e.g. see $[9,10,11,12])$ and that there is a vast literature on rigorous methods to compute solutions of operators defined on Banach spaces (e.g. see $[13,14,15]$ and the references therein).

Based on the previous discussion, the idea consists of computing a numerical approximation $\bar{x}$ of $f(x)=0$ and to demonstrate that close to $\bar{x}$, there exists a genuine solution $x^{*}$ of $f(x)=0$, corresponding to the Floquet normal form of the principal fundamental matrix solution $\Phi(t)$ of (1). However, since the operator $f$ is infinite dimensional, a finite dimensional approximation of $f$ must be introduced in order to compute an approximate solution $\bar{x}$. This is done in Section 2.2. Once $\bar{x}$ is computed, a Newton-like operator $T: \Omega^{s} \rightarrow \Omega^{s}$ defined by $T(x)=x-J f(x)$ is introduced, where $J$ is an injective linear operator which acts as an approximation for $D f(\bar{x})^{-1}$. Since $J$ is injective, the fixed points of $T$ and the zeros of $f$ are in one-to-one correspondence. Denoting by $B(r, s)$ the closed ball of radius $r$ in $\Omega^{s}$, the idea is to consider the ball $B_{\bar{x}}(r, s)=\bar{x}+B(r, s)$ of a priori unknown radius $r$ and to solve for $r$ for which $T: B_{\bar{x}}(r, s) \rightarrow B_{\bar{x}}(r, s)$ is a contraction (see Section 2.3). This is done by deriving a set of sufficient computable conditions in the form of polynomial inequalities, the so-called radii polynomials $\left\{p_{k}(r)\right\}_{k \geq 0}$ (first introduced in [16]), whose successful verification leads to an application of the Contraction Mapping Theorem (CMT) on $T$. More precisely, the radii polynomials $\left\{p_{k}(r)\right\}_{k \geq 0}$ are upper bounds satisfying

$$
\left|(T(\bar{x})-\bar{x})_{k}\right|_{\infty}+\sup _{b_{1}, b_{2} \in B(r, s)}\left|\left[D T\left(\bar{x}+b_{1}\right) b_{2}\right]_{k}\right|_{\infty}-\frac{r}{\omega_{k}^{s}} \leq p_{k}(r), \text { for all } k \geq 0,
$$

where $\frac{r}{\omega_{k}^{s}}$ represents the radius of the $k^{t h}$ component of the ball $B(r, s) \subset \Omega^{s}$, and where $p_{k}(r)$ encloses a defect bound measuring how good the numerical approximation is and a uniform bound on the norm of $D T$ over the entire ball. The construction of the polynomials $\left\{p_{k}(r)\right\}_{k \geq 0}$ is a combination of analytic estimates and interval arithmetic computations. As shown in Theorem 2.6, if one can find $r>0$ such that each radii polynomial is negative, then by the CMT, there exists a unique fixed point of $T$ within $B_{\bar{x}}(r, s)$. It is important to note that only the coefficients of the $p_{k}$ 's are computed, and that the value of the radius $r$ is chosen a posteriori and optimally at the end of the process. This approach is somehow different from the more standard one of fixing a priori the radius $r$ of the ball, of computing the necessary bounds and finally to verify the hypotheses of the CMT by verifying a set of inequalities. The advantage of the radii polynomial approach is twofold. First, most of the estimates are done analytically and generally, hence providing explicit formulas that can give insights into the problems under study. Second, costly computations involving interval arithmetic can be postponed to the very end of the proofs, hence reducing significantly the computational cost (e.g. see [16, 17]). The radii polynomials are introduced in Section 2.3, and we present the explicit bounds in Section 2.4 that lead directly to their construction.

The numerical results reported in this paper are only for the case when the state space is three-dimensional and the nonlinearities of the vector fields are quadratic. These restrictions allows on the one hand more complete visualization and on the other hand allows presenting 
applications in a simple fashion. Increasing the dimension of the systems is straightforward and increasing the degree of the polynomial nonlinearities should also be straightforward, at least theoretically, by applying the analytic estimates of [18]. Let us finally mention that our method should in principle be applicable to any analytic vector fields even though as of now, only polynomial vector fields have been considered.

The paper is organized as follows. In Section 2, we introduce the rigorous computational method to compute Floquet normal forms of fundamental matrix solutions. In Section 3, we demonstrate how to use the information from Floquet normal forms to compute stable and unstable bundles of periodic orbits of vector field and how to determine the linear stability properties of periodic orbits using that information. In Section 4, we combine the ideas of Section 2 and Section 3 to construct rigorously stable and unstable bundles of some periodic orbits of the Lorenz equations (Section 4.1) and of the $\zeta^{3}$-model (Section 4.2). Finally, in Section 4.3, we discuss how to recover a posteriori the Floquet multipliers associated to the periodic orbit $\gamma(t)$, that is the eigenvalues of the monodromy matrix.

\section{Rigorous computation of Floquet normal forms}

In this section, we introduce the rigorous numerical method to compute Floquet normal forms $\Phi(t)=Q(t) e^{R t}$ of fundamental matrix solutions of systems of the form (1). Before doing that, let us fix some notation that is adopted throughout the paper.

Notation

- $\operatorname{Mat}(n, \mathbb{R})$ denotes the space of $n \times n$ matrices with real entries.

- $I_{n}$ is the $n \times n$ identity matrix, $\mathbb{1}_{n}$ is the $n \times n$ matrix whose entries are all 1 .

Let $A, B$ be matrices with entries $A=\left\{a_{i, j}\right\}, B=\left\{b_{i, j}\right\}$ and let $\mathbb{A}=\left(A_{1}, \ldots, A_{n}\right)$, $\mathbb{B}=\left(B_{1}, \ldots, B_{n}\right)$ be vectors of matrices.

- $\|A\|_{\infty}$ is the standard infinity matrix norm: $\|A\|_{\infty}=\max _{i} \sum_{j}\left|a_{i, j}\right|$;

- $|A|=\left\{\left|a_{i, j}\right|\right\}$ is the matrix of absolute values, where $|\cdot|$ denotes both the real and complex absolute value depending wether $a_{i, j}$ is real or complex, and $|A|_{\infty}=\max _{i, j}\left\{\left|a_{i, j}\right|\right\}$. For vectors $|\mathbb{A}|=\left(\left|A_{1}\right|, \ldots,\left|A_{n}\right|\right)$ and $|\mathbb{A}|_{\infty}=\max \left\{\left|A_{1}\right|_{\infty}, \ldots,\left|A_{n}\right|_{\infty}\right\}$

- $\leq_{c w}$ is the component-wise inequality: $A \leq_{c w} B$ means $a_{i, j} \leq b_{i, j}$ for any $i, j$. In case $b$ is a scalar, $A \leq_{c w} b$ means $a_{i, j} \leq b$. In case of vectors $\mathbb{A} \leq_{c w} \mathbb{B}$ and $\mathbb{A} \leq_{c w} b$ extends as $A_{k} \leq_{c w} B_{k}$ and $A_{k} \leq_{c w} b$, for any $k=1 \ldots n$. The same for $\geq_{c w},>_{c w},<_{c w}$;

\subsection{Set-up of the operator equation $f(x)=0$}

As already mentioned in Section 1, the first step is to introduce the nonlinear operator $f$ whose zeros are in one-to-one correspondence with the solutions of (3).

The computation of the Floquet normal form of the principal fundamental matrix solution is done by solving system (3) where the unknowns are the $2 \tau$-periodic matrix-valued function $Q(t)$ and the constant matrix $R$, while the $\tau$-periodic matrix-valued function $A(t)$ is known. Let us consider the expansion of $Q(t)$ in Fourier series

$$
Q(t)=\mathcal{Q}_{0}+\sum_{k \in \mathbb{Z} \backslash\{0\}}\left(\mathcal{Q}_{k, 1}+i \mathcal{Q}_{k, 2}\right) e^{i k \frac{2 \pi}{2 \tau} t},
$$


where the Fourier coefficients $\mathcal{Q}_{0}, \mathcal{Q}_{k, i} \in \operatorname{Mat}(n, \mathbb{R})$ satisfy $\mathcal{Q}_{-k, 1}=\mathcal{Q}_{k, 1}$ and $\mathcal{Q}_{-k, 2}=-\mathcal{Q}_{k, 2}$ for any $k \geq 1$. Being $\tau$-periodic, the matrix function $A(t)$ is also $2 \tau$-periodic and therefore can be expanded as

$$
A(t)=\mathcal{A}_{0}+\sum_{k \in \mathbb{Z} \backslash\{0\}}\left(\mathcal{A}_{k, 1}+i \mathcal{A}_{k, 2}\right) e^{i k \frac{2 \pi}{2 \tau} t},
$$

where $\mathcal{A}_{0}, \mathcal{A}_{k, i} \in \operatorname{Mat}(n, \mathbb{R})$ and $\mathcal{A}_{-k, 1}=\mathcal{A}_{k, 1}$ and $\mathcal{A}_{-k, 2}=-\mathcal{A}_{k, 2}$. In addition, since $A(t)$ is $\tau$-periodic, $\mathcal{A}_{k, 1}=\mathcal{A}_{k, 2}=0$ for $k$ odd and $\mathcal{A}_{2 l, i}=\hat{\mathcal{A}}_{l, i}$ where $\hat{\mathcal{A}}_{l, i}$ is the $l$-th Fourier coefficient of $A(t)$ in the basis $\left\{e^{i k \frac{2 \pi}{\tau} t}\right\}_{k}$. From now on, we use the notation

$$
\mathcal{A}_{k}=\mathcal{A}_{k, 1}+i \mathcal{A}_{k, 2}
$$

to denote the $k^{\text {th }}$ complex Fourier coefficient of $A(t)$, unless it is necessary to separate the real and imaginary part of the Fourier coefficients of $A(t)$.

Substituting the expansions (4) and (5) in (3) results in an equation of the form $F(t)=0$, where $F(t)$ is a $2 \tau$-periodic matrix function. Expanding $F(t)$ in the Fourier basis $\left\{e^{i k \frac{2 \pi}{2 \tau} t}\right\}$, it follows that solving (3) is equivalent to solve for the unknowns

$$
R, \mathcal{Q}_{0} \in \operatorname{Mat}(n, \mathbb{R}) \text { and } \mathcal{Q}_{k}:=\left(\mathcal{Q}_{k, 1}, \mathcal{Q}_{k, 2}\right) \in \operatorname{Mat}(n, \mathbb{R})^{2}
$$

the infinite dimensional algebraic system

$$
\begin{aligned}
& f\left(R, \mathcal{Q}_{0}, \ldots, \mathcal{Q}_{k}, \ldots\right)=0 \\
& f=\left(f_{\star}, f_{0}, f_{1}, \ldots, f_{k}, \ldots\right)
\end{aligned}
$$

defined by

$$
\begin{aligned}
f_{\star} & :=\mathcal{Q}_{0}+2 \sum_{k \geq 1} \mathcal{Q}_{k, 1}-I_{n} \\
f_{0} & :=\mathcal{Q}_{0} R-(\mathcal{A} * \mathcal{Q})_{0} \\
f_{k} & :=\left[\begin{array}{l}
f_{k, 1} \\
f_{k, 2}
\end{array}\right]=\left[\begin{array}{r}
-k \frac{2 \pi}{2 \tau} \mathcal{Q}_{k, 2}+\mathcal{Q}_{k, 1} R-(\mathcal{A} * \mathcal{Q})_{k, 1} \\
k \frac{2 \pi}{2 \tau} \mathcal{Q}_{k, 1}+\mathcal{Q}_{k, 2} R-(\mathcal{A} * \mathcal{Q})_{k, 2}
\end{array}\right], \quad k \geq 1,
\end{aligned}
$$

where $(\mathcal{A} * \mathcal{Q})_{k, 1},(A * \mathcal{Q})_{k, 2}$ denote respectively the real and imaginary part of the convolution

$$
(\mathcal{A} * \mathcal{Q})_{k}:=\sum_{k_{1}+k_{2}=k}\left(\mathcal{A}_{k_{1}, 1}+i \mathcal{A}_{k_{1}, 2}\right)\left(\mathcal{Q}_{k_{2}, 1}+i \mathcal{Q}_{k_{2}, 2}\right)
$$

Since $f_{\star}, f_{0} \in \operatorname{Mat}(n, \mathbb{R})$ and $f_{k} \in \operatorname{Mat}(n, \mathbb{R})^{2}$ for every $k \geq 1$, (6) consists of a system of $n^{2}$ real scalar equations for $f_{\star}=0, n^{2}$ real scalar equations for $f_{0}=0$ and $2 n^{2}$ real scalar equations for each $f_{k}=0(k \geq 1)$. More precisely, $f_{\star}$ represents the initial condition $Q(0)-I_{n}=0$ given by the second component of (3), while $f_{0}$ and $f_{k}$ correspond to the inner product in $L^{2}\left(\left[0, \frac{2 \pi}{2 \tau}\right]\right)$ of $F(t)$ with each element of the Fourier basis $\left\{e^{i k \frac{2 \pi}{2 \tau} t}\right\}$.

Consider the space of unknowns by

$$
X=\left\{x=\left(x_{0}, x_{1}, \ldots, x_{k}, \ldots\right): \begin{array}{l}
x_{0}=\left(R, \mathcal{Q}_{0}\right) \in \operatorname{Mat}(n, \mathbb{R})^{2} \\
x_{k}=\mathcal{Q}_{k}=\left(\mathcal{Q}_{k, 1}, \mathcal{Q}_{k, 2}\right) \in \operatorname{Mat}(n, \mathbb{R})^{2}, k \geq 1
\end{array}\right\} .
$$


Note that $f: X \rightarrow X$. As mentioned already in Section 1, solving $f(x)=0$ is transformed into a fixed point problem equation $T(x)=x$ which is solved using the contraction mapping theorem (CMT). That requires chosing a suitable Banach subspace of $X$ where the investigation of existence of fixed points of $T$ is done. Hence, consider the weights

$$
w_{k}=\left\{\begin{array}{cc}
|k| & k \neq 0 \\
1 & k=0
\end{array}\right.
$$

and for any $x=\left(R, \mathcal{Q}_{0}, \mathcal{Q}_{1,1}, \mathcal{Q}_{1,2}, \ldots, \mathcal{Q}_{k, 1}, \mathcal{Q}_{k, 2}, \ldots\right) \in X$ define the $s$-norm of $x$ by

$$
\|x\|_{s}:=\sup _{k \geq 0}\left\{\left|x_{k}\right|_{\infty} w_{k}^{s}\right\}=\sup \left\{|R|_{\infty},\left|\mathcal{Q}_{0}\right|_{\infty}, \sup _{k \geq 1}\left\{\left|\mathcal{Q}_{k, 1}\right|_{\infty} w_{k}^{s},\left|\mathcal{Q}_{k, 2}\right|_{\infty} w_{k}^{s}\right\}\right\} .
$$

Define $\Omega^{s}$ the space of sequences in $X$ with algebraically decaying tails

$$
\Omega^{s}=\left\{x \in X:\|x\|_{s}<\infty\right\} .
$$

For any $s>0$ the space $\Omega^{s}$ endowed with the $s$-norm is a Banach space and the inclusion $\Omega^{s} \supset \Omega^{s+1}$ holds. The main motivation of working in $\Omega^{s}$ is that smoothness of an integrable function is equivalent to fast decay of its Fourier coefficients. Indeed a periodic function, whose series of Fourier coefficients belong to $\Omega^{s}$, is at least of class $C^{s-1}$. If $A(t)$ is analytic, it follows that the solution $Q(t)$ of system (3) is analytic thus the Fourier coefficients of $Q(t)$ decay faster than any power rate and therefore they belong to $\Omega^{s}$ for any $s$. On the other hand, even a weaker assumption on the function $A(t)$, for instance that the Fourier coefficients $\mathcal{A}_{k}$ decay algebraically with power rate $s$, is sufficient to conclude that the solution $x \in \Omega^{s}$. For all applications presented in this paper, the function $A(t)$ comes from an independent rigorous computation and its regularity is provided via an explicit knowledge of the algebraic decay rate of its Fourier coefficients $\mathcal{A}_{k}$.

Denoting by $\mathcal{A}=\left\{\mathcal{A}_{k}\right\}_{k \geq 0}$ the sequence of complex Fourier coefficients appearing in (5), the $s$-norm of $\mathcal{A}$ is given by

$$
\|\mathcal{A}\|_{s}=\sup _{k \geq 0}\left\{\left|\mathcal{A}_{k}\right|_{\infty} w_{k}^{s}\right\} .
$$

Lemma 2.1. Assume $\|\mathcal{A}\|_{s^{\star}}<\infty$ for $s^{\star} \geq 2$. Then $f$ maps $\Omega^{s}$ in $\Omega^{s-1}$, for any $2 \leq s \leq s^{\star}$.

Proof. Let $2 \leq s \leq s^{\star}$ and suppose $x \in \Omega^{s}$. Then $\left|\mathcal{A}_{k}\right|_{\infty}<C_{1} w_{k}^{-s}$ and, from Lemma 2.1 in [18], $\left|(\mathcal{A} * \mathcal{Q})_{k}\right|_{\infty} \leq \frac{C_{2}}{w_{k}^{s}}$. Thus $\left|f_{k}(x)\right|_{\infty} \leq C_{3} k\left|\mathcal{Q}_{k}\right|_{\infty}+C_{4}\left|\mathcal{Q}_{k}\right|_{\infty}+C_{2} w_{k}^{-s}<C w_{k}^{-s+1}$, for suitable constants $C, C_{i}$. This shows that $f(x) \in \Omega^{s-1}$.

Thus we look for solutions $x=\left(R, \mathcal{Q}_{0}, \mathcal{Q}_{1,1}, \mathcal{Q}_{1,2}, \ldots, \mathcal{Q}_{k, 1}, \mathcal{Q}_{k, 2}, \ldots\right)$ of (6) within $\Omega^{s}$ for some $s \geq 2$. Instead of working directly with $f(x)=0$, we introduce a suitable operator $T: \Omega^{s} \rightarrow \Omega^{s}$ whose fixed points are in one-to-one correspondence with the zeros of $f$, and we aim at proving the existence and local uniqueness of its fixed points by verifying the hypothesis of the CMT. More explicitly, in Section 2.2, we first consider a finite dimensional projection of (6) in a finite dimensional subspace of $\Omega^{s}$ and we compute a numerical solution $\bar{x}$. Afterwards, in Section 2.3, the operator $T: \Omega^{s} \rightarrow \Omega^{s}$ is introduced as a modified Newton operator around $\bar{x}$ in a way that a fixed point $x$ of $T$ corresponds to a genuine solution of $f(x)=0$. The hope is that if $\bar{x}$ is a good enough approximation, then $T$ contracts a small ball $B_{\bar{x}}(r, s)$ of radius $r$ (a priori unknown) around $\bar{x}$, proving the existence of a unique fixed point within $B_{\bar{x}}(r, s)$. Thus the method aims at detecting a value $r$ and proving that the operator $T$ satisfies the hypotheses of the CMT on $B_{\bar{x}}(r, s) \subset \Omega^{s}$. Since the proof is a combination of analysis and computations, the hypotheses of the CMT needs to be verifiable 
on a computer. That can be done by means of the so-called radii polynomials introduced in Section 2.3. The radii polynomial consist of a finite number of polynomial inequalities whose verification is sufficient to conclude that the operator $T$ is a contraction on $B_{\bar{x}}(r, s)$. Their construction is performed in Section 2.4 where we show all the analytical estimates necessary to end up with a finite number of computable conditions.

\section{$2.2 \quad$ Finite dimensional projection}

As mentioned earlier, the fist step involved in the computational method is to consider a finite dimensional projection and to compute an approximate numerical solution of (6).

For $m>1$, consider the finite dimensional space $X^{m}=\prod_{k=1}^{m} \operatorname{Mat}(n, \mathbb{R})^{2}$ and define the projections

$$
\begin{aligned}
\Pi^{m}: X & \rightarrow X^{m} \\
x & \mapsto \Pi^{m}(x)=x^{m}=\left(R, \mathcal{Q}_{0}, \ldots, \mathcal{Q}_{m-1}\right) \\
\Pi_{m}^{\infty}: x & \mapsto\left(\mathcal{Q}_{m}, \mathcal{Q}_{m+1}, \ldots\right)
\end{aligned}
$$

so that $x=\left(x^{m}, \Pi_{m}^{\infty}(x)\right)$. Denote with $0_{m}^{\infty}:=\Pi_{m}^{\infty}(0)$. Moreover let us define the restriction of $f(x)$ on $X^{m}$ as the map

$$
\begin{aligned}
f^{(m)}: X^{m} & \rightarrow X^{m} \\
x^{m} & \mapsto \Pi^{m} f\left(x^{m}, 0_{m}^{\infty}\right) .
\end{aligned}
$$

For any $x \in X$, the sequence $\left(x^{m}, 0_{m}^{\infty}\right) \in X$ and the finite dimensional projection $\Pi^{m}$ applied to $f(x)$ reads as $\Pi^{m} f(x)=\left(f_{\star}, f_{0}, \ldots, f_{m-1}\right)(x)$. Since $X^{m}$ is isomorphic to $\mathbb{R}^{m 2 n^{2}}$, one

has that $f^{(m)}: \mathbb{R}^{m 2 n^{2}} \rightarrow \mathbb{R}^{m 2 n^{2}}$. Suppose that using a Newton-like iterative algorithm, one computed

$$
\bar{x}=\left(\bar{R}, \overline{\mathcal{Q}}_{0}, \ldots, \overline{\mathcal{Q}}_{m-1}\right)
$$

an approximate zero of $f^{(m)}$, that is $f^{(m)}(\bar{x}) \approx 0$. For sake of simplicity of the presentation, the same notation $\bar{x}$ is used to identify the vector $\bar{x} \in X^{m}$ defined in (12) and the sequence $\left(\bar{x}, 0_{m}^{\infty}\right) \in X$. As already mentioned at the end of Section 2.1, the idea is to consider a ball $B_{\bar{x}}(r, s) \in \Omega^{s}$ centered at the approximate solution $\bar{x}$ and to show the existence of a contraction mapping $T$ acting on $B_{\bar{x}}(r, s)$. Hence, let us now introduce the fixed point operator $T$.

\subsection{The fixed point operator $T$ and the radii polynomials}

In this section, we reformulate the problem of studying the zeros of $f(x)$ by establishing an equivalent fixed point problem $T(x)=x$ in $\Omega^{s}$, and we introduce the notion of the so-called radii polynomials, which provide an efficient means of finding a set on which $T$ is a contraction. More precisely, the operator $T$ is defined as $T(x)=x-J f(x)$, that is as a Newton-like operator which depends on an approximation $J$ of $D f(\bar{x})^{-1}$. The advantage of considering such approach is that if $J$ is a good enough approximation of the inverse of $D f(\bar{x})$, then $T$ has good chances of being a contraction on a small ball centered at $\bar{x}$. Hence, the first goal of this section is to define $J$. In order to do so, we begin by introducing $J^{\dagger}$ (see (19)) an approximation for $D f(\bar{x})$ that we then use to construct, with the help of the computer, the operator $J$ introduced in (20).

In order to write explicitly the action of $J$ on $\Omega^{s}$, it is convenient to represent matrices in $\operatorname{Mat}(n, \mathbb{R})$ as vectors in $\mathbb{R}^{n^{2}}$, and to extend this representation to get an isomorphism 
between the space of vectors of $N$ matrices $\operatorname{Mat}(n, \mathbb{R})$ to the space $\mathbb{R}^{N n^{2}}$. With this construction, note that $X^{m}$ is isomorphic to $\mathbb{R}^{2 m n^{2}}$.

According to the chosen representation, given a vector $V=\left[v_{1}, \ldots, v_{N 2 n^{2}}\right] \in \mathbb{R}^{2 n^{2} N}$ we denote by $V_{k} \in \mathbb{R}^{2 n^{2}}$ the vector with $2 n^{2}$ components $V_{k}=\left[v_{k 2 n^{2}+1}, v_{k 2 n^{2}+2}, \ldots, v_{2 n^{2}(k+1)}\right]$. This choice of notation is motivates as follows. Suppose that $V$ is the vector representation of the sequence $x=\left(R, \mathcal{Q}_{0}, \mathcal{Q}_{1}, \ldots, \mathcal{Q}_{N-1}\right) \in X^{N}$, then $V_{k}$ collects the entries of $\left(R, \mathcal{Q}_{0}\right)$ for $k=0$ and of $\mathcal{Q}_{k}=\left(\mathcal{Q}_{k, 1}, \mathcal{Q}_{k, 2}\right)$ for $k \geq 1$.

Denote by $D f^{(m)}$ the Jacobian of $f^{(m)}$ with respect to $x^{m}$ evaluated at $\bar{x}$, that is

$$
D f^{(m)}:=D f^{(m)}(\bar{x})=\frac{\partial\left(f_{\star}, f_{0}, f_{1}, \ldots, f_{m-1}\right)}{\partial\left(R, \mathcal{Q}_{0}, \ldots, \mathcal{Q}_{m-1}\right)}(\bar{x}) \in \operatorname{Mat}\left(2 n^{2} m, \mathbb{R}\right) .
$$

More explicitely,

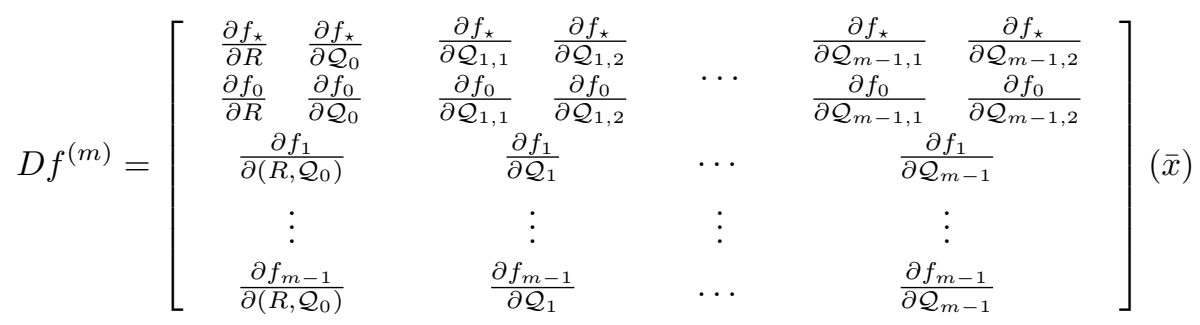

where for $k, j=1, \ldots, m-1$,

$$
\frac{\partial f_{k}}{\partial\left(R, \mathcal{Q}_{0}\right)}=\left[\begin{array}{cc}
\frac{\partial f_{k, 1}}{\partial R} & \frac{\partial f_{k, 1}}{\partial \mathcal{Q}_{0}} \\
\frac{\partial f_{k, 2}}{\partial R} & \frac{\partial f_{k, 2}}{\partial \mathcal{Q}_{0}}
\end{array}\right], \quad \frac{\partial f_{k}}{\partial \mathcal{Q}_{j}}=\left[\begin{array}{cc}
\frac{\partial f_{k, 1}}{\partial \mathcal{Q}_{j, 1}} & \frac{\partial f_{k, 1}}{\partial \mathcal{Q}_{j, 2}} \\
\frac{\partial f_{k, 2}}{\partial \mathcal{Q}_{j, 1}} & \frac{\partial f_{k, 2}}{\partial \mathcal{Q}_{j, 2}}
\end{array}\right],
$$

and each $\frac{\partial f_{k, i}}{\partial \mathcal{Q}_{j, l}} \in \operatorname{Mat}\left(n^{2}, \mathbb{R}\right)$ denotes the Jacobian matrix of the components of $f_{k, i}$ with respect to the components of $\mathcal{Q}_{j, l}$. Moreover, for $k \geq m$, define

$$
\Lambda_{k}:=\frac{\partial f_{k}}{\partial \mathcal{Q}_{k}}(\bar{x}) \in \operatorname{Mat}\left(2 n^{2}, \mathbb{R}\right) .
$$

Lemma 2.2. Recall (5) and (9), and assume that $\|\mathcal{A}\|_{s}<\infty$ for some $s \geq 2$. Then there exists $K \in \mathbb{N}$ such that for any $k \geq K$ the linear operator $\Lambda_{k}$ is invertible. Moreover, for any $M \geq K$ there exists a constant $C_{\Lambda}=C_{\Lambda}(M)$ such that

$$
\left\|\Lambda_{k}^{-1}\right\|_{\infty} \leq \frac{C_{\Lambda}}{k}, \text { for } k \geq M
$$

Proof. Given an index $k$, the real and imaginary parts of $(\mathcal{A} * \mathcal{Q})_{k}$ can be written as

$$
\begin{aligned}
& (\mathcal{A} * \mathcal{Q})_{k, 1}=\left(\mathcal{A}_{0}+\mathcal{A}_{2 k, 1}\right) \mathcal{Q}_{k, 1}+\mathcal{A}_{2 k, 2} \mathcal{Q}_{k, 2}+W_{1}, \\
& (A * Q)_{k, 2}=\mathcal{A}_{2 k, 2} \mathcal{Q}_{k, 1}+\left(\mathcal{A}_{0}-\mathcal{A}_{2 k, 1}\right) \mathcal{Q}_{k, 2}+W_{2},
\end{aligned}
$$

where $W_{1}$ and $W_{2}$ do not depend on $\mathcal{Q}_{k, 1}$ and $\mathcal{Q}_{k, 2}$. Thus, looking at the definition of $f_{k}$ in (7), it follows that $\Lambda_{k}$ is of the form

$$
\Lambda_{k}=\left[\begin{array}{cc}
\lambda_{1,1} & -k \frac{2 \pi}{2 \tau} I_{n^{2}}+\lambda_{1,2} \\
k \frac{2 \pi}{2 \tau} I_{n^{2}}+\lambda_{2,1} & \lambda_{2,2}
\end{array}\right],
$$


where the entries of $\lambda_{1,1}$ and $\lambda_{2,2}$ satisfy $\left|\lambda_{1,1}\right|_{\infty},\left|\lambda_{2,2}\right|_{\infty}<|\bar{R}|_{\infty}+\left|\mathcal{A}_{0}\right|_{\infty}+\left|\mathcal{A}_{2 k}\right|_{\infty}$ and $\lambda_{2,1}=\lambda_{1,2}$ satisfy $\left|\lambda_{2,1}\right|_{\infty}=\left|\lambda_{1,2}\right|_{\infty}<\left|\mathcal{A}_{2 k}\right|_{\infty}$. By a row permutation, the invertibility of $\Lambda_{k}$ is equivalent to the invertibility of

$$
\hat{\Lambda}_{k}:=\left[\begin{array}{cc}
k \frac{2 \pi}{2 \tau} I_{n^{2}}+\lambda_{2,1} & \lambda_{2,2} \\
\lambda_{1,1} & -k \frac{2 \pi}{2 \tau} I_{n^{2}}+\lambda_{1,2}
\end{array}\right] .
$$

Since $\left|\lambda_{1,1}\right|_{\infty},\left|\lambda_{2,2}\right|_{\infty}<|\bar{R}|_{\infty}+\left|\mathcal{A}_{0}\right|_{\infty}+\left|\mathcal{A}_{2 k}\right|_{\infty}$ and $\left|\lambda_{1,2}\right|_{\infty}<\left|\mathcal{A}_{2 k}\right|_{\infty}$, the hypothesis $\|\mathcal{A}\|_{s}<\infty$ implies that the $\left|\lambda_{i, j}\right|_{\infty}$ are uniformly bounded in $k$ and $\left|\lambda_{1,2}\right|_{\infty}$ is decreasing as $k$ increases. Thus there exists $K$ such that for any $k \geq K$ the matrix $\hat{\Lambda}_{k}$ is diagonally dominant, i.e. $\left|\hat{\Lambda}_{k}(i, i)\right|>\sum_{j \neq i}\left|\hat{\Lambda}_{k}(i, j)\right|$ for any $i=1, \ldots, 2 n^{2}$. This is enough to conclude that $\hat{\Lambda}_{k}$ is invertible for any $k \geq K$. Moreover, it follows (e.g. see from [19]) that

$$
\left\|\hat{\Lambda}_{k}^{-1}\right\|_{\infty} \leq \max _{i}\left\{\frac{1}{\left|\hat{\Lambda}_{k}(i, i)\right|-\sum_{j \neq i}\left|\hat{\Lambda}_{k}(i, j)\right|}\right\} .
$$

Therefore, for any $M \geq K$, there exists a constant $C_{\Lambda}=C_{\Lambda}(M)$ such that

$$
\left\|\Lambda_{k}^{-1}\right\|_{\infty}=\left\|\hat{\Lambda}_{k}^{-1}\right\|_{\infty} \leq \frac{C_{\Lambda}}{k}, \quad \forall k \geq M .
$$

A general construction of the constant $C_{\Lambda}$ is done in the Appendix in Section 6.1 and its formula is given by (67). Remark that this construction depends on the uniform bound of the matrices $\mathcal{A}_{2 k}$ for $k \geq M$. For sake of generality, we considered the weakest hypothesis $\|\mathcal{A}\|_{s^{*}}<\infty$, yielding the formula (67). However, more information about the behavior of $\mathcal{A}_{2 k}$ allows constructing a sharper bound $C_{\Lambda}$. An example of this is given in Section 4.1 in the context of the Lorenz equations, where a sharper bound for $C_{\Lambda}$ is provided.

Recalling (13) and (14), one has that if the finite dimensional projection $m$ is taken large enough, then the action of $D f(\bar{x})$ should in principle be governed by $D f^{(m)}$ and $\left\{\Lambda_{k}\right\}_{k \geq m}$. Indeed, the off diagonal terms of the derivative $D f(\bar{x})$ are given by $\frac{\partial f_{j}}{\partial \mathcal{Q}_{i}}$ and they depend on $\mathcal{A}_{j-i}$ which decreases to zero as $|j-i|$ increases. However, since the phase condition $f_{\star}(x)$ given by the first component of $(7)$ acts linearly on $x$, a better finite dimensional approximation of $D f(\bar{x})$ is given by $L_{M}: X^{M} \rightarrow X^{M}$ defined by

$$
L_{M}=\left[\begin{array}{c|ccc}
D f^{(m)} & \frac{\partial f_{\star}}{\partial Q_{m}}(\bar{x}) & \ldots & \frac{\partial f_{\star}}{\partial Q_{M-1}}(\bar{x}) \\
& 0 & \ldots & 0 \\
& 0 & \ldots & 0 \\
\hline & \Lambda_{m} & & \\
& & \ddots & \\
& & & \Lambda_{M-1}
\end{array}\right],
$$

for a given $M>m$. Consider a linear operator $J^{\dagger}: X \rightarrow X$ acting as an approximation for $D f(\bar{x})$ defined, given $x \in X$, by

$$
\left(J^{\dagger} x\right)_{k}:= \begin{cases}\left(L_{M} \cdot x^{M}\right)_{k} & k=0, \ldots, M-1 \\ \Lambda_{k} x_{k}, & k \geq M\end{cases}
$$

As mentioned above, the operator $J^{\dagger}$ is now used to construct the operator $J$. First we numerically compute an invertible matrix $J_{m} \in \operatorname{Mat}\left(2 n^{2} m, \mathbb{R}\right)$ such that $J_{m} \cdot D f^{(m)} \approx I_{m}$ 
and we introduce an invertible linear operator $J_{M}: X^{M} \rightarrow X^{M}$

$$
J_{M}:=\left[\begin{array}{l|lll}
J_{m} & \multicolumn{3}{|c}{\mathcal{L}} \\
& & & \\
\hline & \left(\Lambda_{m}\right)^{-1} & & \\
& & \ddots & \\
& & & \left(\Lambda_{M-1}\right)^{-1}
\end{array}\right], \text { such that } J_{M} \cdot L_{M} \approx I_{M} .
$$

By straightforward calculations, the matrix $\mathcal{L}$ can be easily constructed combining the matrix $J_{m}$ with the matrices $\left\{\left(\Lambda_{k}\right)^{-1}, \frac{\partial f_{\star}}{\partial Q_{k}}(\bar{x})\right\}_{k=m}^{M-1}$. More explicitely, writing

$$
\mathcal{L}=\left[\begin{array}{l|l|l|l}
\mathcal{L}_{m} & \mathcal{L}_{m+1} & \ldots & \mathcal{L}_{M-1}
\end{array}\right], \quad \mathcal{L}_{k} \in \mathbb{R}^{\left(2 n^{2} m\right) \times\left(2 n^{2}\right)}
$$

we can compute $\mathcal{L}_{k}=-2\left(J_{m}\right)^{\left[1 \ldots n^{2}\right]} \cdot\left(\Lambda_{m}^{-1}\right)_{\left[1, \ldots, n^{2}\right]}$, where the notation $A^{[1, \ldots, k]}$, (respectively $\left.A_{[1, \ldots, k]}\right)$ denotes the submatrix of $A$ given by the first $k$ columns (respectively rows) of $A$. The constant -2 in $\mathcal{L}_{k}$ comes from the fact that $\frac{\partial f_{*}}{\partial \mathcal{Q}_{k, 1}}=2 I_{n}$.

We are now ready to introduce the operator $J$ which defines the fixed point operator $T$. Consider $K$ the constant introduced in Lemma 2.2, the finite dimensional projection $m>K$ and the computational parameter $M>m$. We define $J: X \rightarrow X$ as the formal diagonal concatenation of $J_{M}$ and the sequence $\Lambda_{k}^{-1}$, that is, given $x \in X$,

$$
(J x)_{k}:=\left\{\begin{array}{cl}
\left(J_{M} x^{M}\right)_{k} & k=0, \ldots, M-1 \\
\Lambda_{k}^{-1} x_{k} & k \geq M .
\end{array}\right.
$$

We can finally use the definition of $J$ in (20) to define the operator $T: X \rightarrow X$ by

$$
T(x):=x-J f(x)
$$

Now that an approximation $J$ for the inverse of $D f(\bar{x})$ has been chosen and that the fixed point operator $T$ has been defined, let us demonstrate some properties about $T$.

Lemma 2.3. Recall (5) and (9), and assume that $\|\mathcal{A}\|_{s^{\star}}<\infty$ for $s^{\star} \geq 2$. Then for any $2 \leq s \leq s^{\star}, T: \Omega^{s} \rightarrow \Omega^{s}$ and solutions of $T(x)=x$ correspond to solutions of $f(x)=0$.

Proof. Recalling (8), the linear operator $J$ defined in (20) maps $\Omega^{s-1}$ to $\Omega^{s}$. Indeed, considering $k \geq M$ and using the fact that $M>m>K$, Lemma 2.2 can be used to conclude that $\Lambda_{k}$ given by (14) is invertible and that $\left|(J x)_{k}\right|_{\infty}=\left|\Lambda_{k}^{-1} x_{k}\right|_{\infty} \leq\left.\left.\left\|\Lambda_{k}^{-1}\right\|_{\infty}\right|_{x_{k}}\right|_{\infty} \leq$ $\frac{C_{\Lambda}}{k} \frac{\|x\|_{s-1}}{w_{k}^{s-1}}<\frac{C}{w_{k}^{s}}$, for a positive constants $C$. Since $f: \Omega^{s} \rightarrow \Omega^{s-1}$ by Lemma 2.1 , we conclude that $T: \Omega^{s} \rightarrow \Omega^{s}$. Since $J_{M}$ is invertible by assumption and $\Lambda_{k}$ is invertible for all $k \geq m>K$, it follows that the linear operator $J$ is invertible and therefore that the fixed points of $T$ are in one-to-one correspondence with the zeros of $f$.

Now that $\bar{x}$ and $T$ are fixed once and for all, the only remaining variable to be found is the radius $r$ of the ball $B_{\bar{x}}(r, s)$. In order to find a successful $r$, we construct a finite number of polynomial inequalities, the so-called radii polynomials $\left\{p_{k}(r)\right\}_{k \geq 0}$, which provide sufficient and efficient computable conditions to have that $T: B_{\bar{x}}(r, s) \rightarrow B_{\bar{x}}(r, s)$ is a contraction. As mentioned earlier, the radii polynomials are a priori conditions that are derived using 
analytic estimates and interval arithmetic, and once their construction is done theoretically, they are used to solve for the optimal radius of the ball $B_{\bar{x}}(r, s)$.

Before proceeding further, let us compare briefly the radii polynomials approach with some more standard rigorous computational a posteriori methods. First, note that our approach is similar to the Krawczyk operator approach [20, 21] and to the method developed by Yamamoto in [10]. However, these methods consist of applying directly the operator to interval vectors (also in the form of small sets centered at a numerical approximation) and then attempt to verify a posteriori the hypotheses of a contraction mapping argument. Second, let us emphasize the differences between our approach and the a posteriori approaches based on verifying the hypotheses of the following standard CMT (e.g. see [13]).

Theorem 2.4 (Standard CMT). If there exist two positive numbers $\rho<1$ and $\varepsilon<(1-\rho) r$ such that $\|T(\bar{x})-\bar{x}\|_{s} \leq \varepsilon$ and $\sup _{x \in B_{\bar{x}}(r, s)}\|D T(x)\|_{s} \leq \rho$, then there is a unique fixed point $x^{*}$ of $T$ in $B_{\bar{x}}(r, s)$ and $\left\|x^{*}-\bar{x}\right\|_{s}<\varepsilon /(1-\rho)$.

A common way to verify the hypotheses of Theorem 2.4 is to fix the radius $r$ of the ball $B_{\bar{x}}(r, s)$, to compute the bounds $\rho$ and $\varepsilon$, and to verify a posteriori that $\rho<1$ and $\varepsilon<(1-\rho) r$. If $\rho$ and $\varepsilon$ do not satisfy these bounds, one can either increase or decrease the radius $r$, and to start over the computations. Our approach is different in the sense that first, we compute an upper bound for $\|T(\bar{x})-\bar{x}\|_{s}$ by computing an upper bound $Y_{k}$ for each component $\left|[T(\bar{x})-\bar{x}]_{k}\right|$ and second, we compute an upper bound for $\sup _{x \in B_{\bar{x}}(r, s)}\|D T(x)\|_{s}$ by expanding each component $\sup _{x_{1}, x_{2} \in B(r, s)} D T_{k}\left(x_{1}+\bar{x}\right) x_{2}$ as a polynomial $Z_{k}(r)$ in the $a$ priori unknown radius $r$. The component-wise resulting bounds are finally used to construct each radii polynomials $p_{k}(r)=Y_{k}+Z_{k}(r)-\frac{r}{w_{k}^{s}}$. Afterward, we solve for $r>0$ such that $p_{k}(r)<0$. Recalling the norm in $\Omega^{s}$ given by (8), the corresponding $\varepsilon$ and $\rho$ from Theorem 2.4 are given by $\varepsilon=\|Y\|_{s}$ and $\rho=\frac{\|Z\|_{s}}{r}$. See the similarities with Theorem 2.5.

Let us now be more explicit about the bounds $Y$ and $Z$. Suppose there exist two matrices sequences

$$
Y=\left(Y_{0}, Y_{1}, \ldots Y_{k}, \ldots\right), \quad Z(r)=\left(Z_{0}(r), Z_{1}(r), \ldots Z_{k}(r), \ldots\right), \quad Y, Z(r) \in X
$$

such that

$$
\left|(T(\bar{x})-\bar{x})_{k}\right| \leq_{c w} Y_{k}, \quad \sup _{b_{1}, b_{2} \in B(r, s)}\left|\left[D T\left(\bar{x}+b_{1}\right) b_{2}\right]_{k}\right| \leq_{c w} Z_{k}(r), \quad \forall k \geq 0 .
$$

Theorem 2.5. Fix $s \geq 2$ and let $Y$ and $Z$ be defined as in (22). If there exists $r>0$ such that $\|Y+Z\|_{s}<r$, then the operator $T$ maps $B_{\bar{x}}(r, s)$ into itself and $T: B_{\bar{x}}(r, s) \rightarrow B_{\bar{x}}(r, s)$ is a contraction. Thus, by the contraction mapping theorem, there exists an unique $x^{*} \in$ $B_{\bar{x}}(r, s)$ solution of $T\left(x^{*}\right)=x^{*}$ and therefore solution of $f\left(x^{*}\right)=0$.

Proof. Two statements need to be proved:

i) $T\left(B_{\bar{x}}(r, s)\right) \subset B_{\bar{x}}(r, s)$, that is $\|T(x)-\bar{x}\|_{s}<r$ for all $x \in B_{\bar{x}}(r, s)$,

ii) $T$ is a contraction, that is

$$
\exists \kappa \in(0,1) \text { such that } \forall x, y \in B_{\bar{x}}(r, s),\|T(x)-T(y)\|_{s} \leq \kappa\|x-y\|_{s} .
$$

For a given $k \geq 0$ and any $x, y \in B_{\bar{x}}(r)$, the Mean Value Theorem implies that

$$
T_{k}(x)-T_{k}(y)=D T_{k}(z)(x-y)
$$


for some $z \in\{t x+(1-t) y: t \in[0,1]\} \subset B_{\bar{x}}(r, s)$. Note that $r \frac{(x-y)}{\|x-y\|_{s}} \in B(r, s)$. Thus from the inequalities in (22),

$$
\left|T_{k}(x)-T_{k}(y)\right|=\left|D T_{k}(z) \frac{r(x-y)}{\|x-y\|_{s}}\right| \frac{1}{r}\|x-y\|_{s} \leq_{c w} \frac{Z_{k}(r)}{r}\|x-y\|_{s} .
$$

The triangular inequality applied component-wise gives

$$
\left|T_{k}(x)-\bar{x}_{k}\right| \leq_{c w}\left|T_{k}(x)-T_{k}(\bar{x})\right|+\left|T_{k}(\bar{x})-\bar{x}_{k}\right| \leq_{c w} Y_{k}+Z_{k}(r)
$$

and hence

$$
\left|T_{k}(x)-\bar{x}_{k}\right|_{\infty} \leq\left|Y_{k}+Z_{k}(r)\right|_{\infty} .
$$

Therefore for any $x \in B_{\bar{x}}(r, s)$

$$
\|T(x)-\bar{x}\|_{s}=\sup _{k \geq 0}\left\{\left|T_{k}(x)-\bar{x}_{k}\right|_{\infty} w_{k}^{s}\right\} \leq \sup _{k \geq 0}\left\{\left|Y_{k}+Z_{k}(r)\right|_{\infty} w_{k}^{s}\right\}=\|Y+Z(r)\|_{s}<r .
$$

This proves $i)$. By (23), for any $x, y \in B_{\bar{x}}(r, s),\left|T_{k}(x)-T_{k}(y)\right|_{\infty} \leq \frac{\left|Z_{k}(r)\right|_{\infty}}{r}\|x-y\|_{s}$. Thus,

$$
\|T(x)-T(y)\|_{s} \leq \frac{\|Z(r)\|_{s}}{r}\|x-y\|_{s} .
$$

Since all the entries of $Y_{k}$ and $Z_{k}(r)$ are non negative, $\left|Z_{k}(r)\right|_{\infty} \leq\left|Y_{k}+Z_{k}(r)\right|_{\infty}$ and $\|Z(r)\|_{s} \leq\|Y+Z(r)\|_{s}<r$. Defining

$$
\kappa:=\frac{\|Z(r)\|_{s}}{r} \in(0,1),
$$

we conclude that $i i$ ) holds. An application of the contraction mapping theorem on the Banach space $B_{\bar{x}}(r, s)$ gives the existence and unicity of a solution $x^{*}$ of the equation $T(x)=$ $x$ in $B_{\bar{x}}(r, s)$ and, from Lemma 2.3, of a solution of $f(x)=0$.

One gets from Theorem 2.5 that a sufficient condition for the existence of a solution of our problem is that the bounds $Y, Z(r)$ satisfying (22) also satisfy $\|Y+Z(r)\|_{s}<r$ for some $r$. We treat the last condition as an inequality to be solved in $r$ and we introduce the radii polynomials as a set of $r$-dependent polynomials $p_{k}(r)$ with the property that a common solution $r^{*}$ of $p_{k}\left(r^{*}\right)<0$ implies that $\left\|Y+Z\left(r^{*}\right)\right\|_{s}<r^{*}$. Each component of the full norm inequality $\|Y+Z(r)\|_{s}<r$ can be written as

$$
\left|Y_{k}+Z_{k}(r)\right|_{\infty}-\frac{r}{w_{k}^{s}}<0, \quad \forall k \geq 0,
$$

which is a system of infinitely many inequalities. In order to reduce (24) to a finite number of inequalities, suppose that, for a given $M$, there exist $Y_{M}$ and $Z_{M}(r)$ such that

$$
\left|(T(\bar{x})-\bar{x})_{k}\right|_{\infty} \leq \frac{M^{s}}{k^{s}} Y_{M}, \quad \sup _{b_{1}, b_{2} \in B(r, s)}\left|\left[D T\left(\bar{x}+b_{1}\right) b_{2}\right]_{k}\right|_{\infty} \leq \frac{M^{s}}{k^{s}} Z_{M}(r), \quad \forall k \geq M .
$$

We can finally define the set of $M+1$ radii polynomials as follows.

Definition 1. The radii polynomials are defined as

$$
\begin{aligned}
& p_{k}(r):=Y_{k}+Z_{k}(r)-\frac{r}{w_{k}^{s}}\left(\mathbb{1}_{n}, \mathbb{1}_{n}\right), \quad k=0, \ldots, M-1 \\
& p_{M}:=Y_{M}+Z_{M}(r)-\frac{r}{w_{M}^{s}} .
\end{aligned}
$$


Theorem 2.6. Let $M>m>K>0$ and suppose $Y=\left\{Y_{k}\right\}_{k \geq 0}, Z(r)=\left\{Z_{k}(r)\right\}_{k \geq 0}$ are sequences of matrices such that $Y_{k}, Z_{k}(r)$ satisfy $(22)$ for $k=0, \ldots, M-1$ and

$$
Y_{k}:=\frac{M^{s}}{k^{s}} Y_{M}\left(\mathbb{1}_{n}, \mathbb{1}_{n}\right), \quad Z_{k}(r):=\frac{M^{s}}{k^{s}} Z_{M}(r)\left(\mathbb{1}_{n}, \mathbb{1}_{n}\right), \quad \forall k \geq M
$$

where $Y_{M}, Z_{M}(r)$ satisfy (25). If there exists $r>0$ such that $p_{k}(r)<_{c w} 0$ for all $k=$ $0, \ldots, M$, then there exists a unique $x^{*} \in B_{\bar{x}}(r, s)$ such that $T\left(x^{*}\right)=x^{*}$ and $f\left(x^{*}\right)=0$.

Proof. Condition (25) implies that $Y_{k}, Z_{k}(r)$ satisfy (22) for all $k \geq M$. By definition of $Y_{k} \geq_{c w} 0, Z_{k}(r) \geq_{c w} 0$, the relation $p_{k}(r)<_{c w} 0$ implies that $\left|Y_{k}+Z_{k}(r)\right|_{\infty}<\frac{r}{w_{k}^{s}}$ for $k=0, \ldots, M-1$. Moreover, from the hypothesis $p_{M}(r)<0$,

$$
\left|Y_{k}+Z_{k}(r)\right|_{\infty}=\frac{M^{s}}{k^{s}}\left(Y_{M}+Z_{M}\right)<\frac{M^{s}}{k^{s}} \frac{r}{M^{s}}=\frac{r}{w_{k}^{s}}, \quad \forall k \geq M
$$

Hence

$$
\|Y+Z\|_{s}=\sup _{k \geq 0}\left\{\left|Y_{k}+Z_{k}\right| w_{k}^{s}\right\}<r
$$

and the result follows from Theorem 2.5.

The strategy to compute rigorously Floquet normal forms of fundamental matrix solutions is then to construct the radii polynomials of Definition 1 and then to attempt to verify the hypotheses of Theorem 2.6. In order to construct the radii polynomials, we need to compute the bounds $Y$ and $Z(r)$ satisfying (22) and (25).

\subsection{Construction of the bounds $Y, Z(r)$}

This section is devoted to the construction of the matrices $Y_{k}, Z_{k}(r)$ for $k=0, \ldots, M-1$ satisfying (22), and of the asymptotic bounds $Y_{M}, Z_{M}(r)$ satisfying (25). This construction provides the complete description of the radii polynomials introduced in Definition 1 . With the aim of remaining as general as possible, the only constraint we assume on the $\tau$-periodic function $A(t)$ is that the vector of Fourier coefficients $\mathcal{A}$ given in (5) satisfies $\|\mathcal{A}\|_{s^{\star}}<\infty$ for $s^{\star} \geq 2$. Nevertheless, further information on the coefficients $\mathcal{A}_{k}$ may be useful to get sharper analytic estimates, as one shall see in the applications of Section 4 .

In what follows, the growth rate parameter $s$ has been fixed so that $2 \leq s \leq s^{\star}$, the finite dimensional parameter $m$ has been chosen so that $m>K$, where $K$ is a lower bound given in Lemma 2.2 and the computational parameter $M$ has been chosen so that $M>m$. Moreover, assume that a numerical solution $\bar{x}$ of the form (12) of the finite dimensional problem $f^{(m)}(x)=0$ has been computed and that $\Lambda_{k}^{-1}$, the inverse of the matrices defined in (14), for $k=m, \ldots, M-1$ is known. The last computation can be done analytically or, if not possible, using rigorous numerics.

\subsubsection{The bound $Y$}

Recalling the definition of the Newton-like operator $T$ in $(21)$, one has that $T(\bar{x})-\bar{x}=$ $-J f(\bar{x})$, and recalling the definition of $J$ in (20), we can define $Y_{k}$ as

$$
Y_{k}=\left|\left(J_{M} f^{(M)}(\bar{x})\right)_{k}\right|, \quad k=0, \ldots, M-1 .
$$


Recalling (25), the tail bound $Y_{M}$ has to be chosen so that $Y_{M} \frac{M^{s}}{k^{s}}>\left|\Lambda_{k}^{-1} f_{k}(\bar{x})\right|_{\infty}$, for any $k \geq M$. To define such bound, we use the fact that $\left|\Lambda_{k}^{-1} f_{k}(\bar{x})\right|_{\infty} \leq\left\|\Lambda_{k}^{-1}\right\|_{\infty}\left|f_{k}(\bar{x})\right|_{\infty}$. Since $\overline{\mathcal{Q}}_{k, 1}=\overline{\mathcal{Q}}_{k, 2}=0$ for any $k \geq m$, it follows that

$$
f_{k}(\bar{x})=\left[\begin{array}{c}
-(\mathcal{A} * \mathcal{Q})_{k, 1} \\
-(\mathcal{A} * \mathcal{Q})_{k, 2}
\end{array}\right]=\sum_{\substack{k_{1}+k_{2}=k \\
\left|k_{2}\right|<m}}\left[\begin{array}{c}
-\operatorname{Re}\left(\mathcal{A}_{k_{1}}\left(\overline{\mathcal{Q}}_{k_{2}, 1}+i \overline{\mathcal{Q}}_{k_{2}, 2}\right)\right) \\
-\operatorname{Im}\left(\mathcal{A}_{k_{1}}\left(\overline{\mathcal{Q}}_{k_{2}, 1}+i \overline{\mathcal{Q}}_{k_{2}, 2}\right)\right)
\end{array}\right], \quad \forall k \geq M .
$$

Now, using the fact that $\left|\mathcal{A}_{k}\right|_{\infty} \leq\|\mathcal{A}\|_{s^{\star}} w_{k}^{-s^{\star}}$, both $\left|f_{k, 1}(\bar{x})\right|$ and $\left|f_{k, 2}(\bar{x})\right|$ are componentwise bounded by

$$
\begin{aligned}
\left|\sum_{\substack{k_{1}+k_{2}=k \\
\left|k_{2}\right|<m}} \mathcal{A}_{k_{1}}\left(\overline{\mathcal{Q}}_{k_{2}, 1}+i \overline{\mathcal{Q}}_{k_{2}, 2}\right)\right| & \leq \sum_{\substack{k_{1}+k_{2}=k \\
\left|k_{2}\right|<m}}\left|\mathcal{A}_{k_{1}}\right|\left|\overline{\mathcal{Q}}_{k_{2}, 1}+i \overline{\mathcal{Q}}_{k_{2}, 2}\right| \\
& \leq{ }_{c w}\left|\mathcal{A}_{k}\right|\left|\overline{\mathcal{Q}}_{0}\right|+\sum_{l=1}^{m-1}\left(\left|\mathcal{A}_{k-l}\right|+\left|\mathcal{A}_{k+l}\right|\right)\left|\overline{\mathcal{Q}}_{l, 1}+i \overline{\mathcal{Q}}_{l, 2}\right| \\
\leq{ }_{c w} \frac{\|\mathcal{A}\|_{s^{\star}}}{w_{k}^{s}} & {\left[\frac{w_{k}^{s}}{w_{k}^{s^{\star}}} \mathbb{1}_{n}\left|\overline{\mathcal{Q}}_{0}\right|+\sum_{l=1}^{m-1} w_{k}^{s}\left(\frac{1}{w_{k+l}^{s^{\star}}}+\frac{1}{w_{k-l}^{s^{\star}}}\right) \mathbb{1}_{n}\left|\overline{\mathcal{Q}}_{l, 1}+i \overline{\mathcal{Q}}_{l, 2}\right|\right] . }
\end{aligned}
$$

Since $\frac{w_{k}^{s}}{w_{k}^{s^{\star}}} \leq 1$ and $w_{k}^{s}\left(\frac{1}{w_{k+l}^{s^{\star}}}+\frac{1}{w_{k-l}^{s^{\star}}}\right) \leq 1+\left(1-\frac{l}{M}\right)^{-s}$ for any $k \geq M$, it follows that

$$
\left|f_{k, i}(\bar{x})\right|_{\infty} \leq k^{-s}\|\mathcal{A}\|_{s^{\star}}|W|_{\infty}, \quad \text { for } k \geq M
$$

where

$$
W=\mathbb{1}_{n}\left|\overline{\mathcal{Q}}_{0}\right|+\sum_{l=1}^{m-1}\left(1+\left(1-\frac{l}{M}\right)^{-s}\right) \mathbb{1}_{n}\left|\overline{\mathcal{Q}}_{l, 1}+i \overline{\mathcal{Q}}_{l, 2}\right| .
$$

From Lemma 2.2, there exists $C_{\Lambda}$ such that $\left\|\Lambda_{k}^{-1}\right\|_{\infty} \leq \frac{C_{\Lambda}}{M}$ for $k \geq M$. Finally, we let

$$
Y_{M}:=\frac{1}{M^{s+1}}\|\mathcal{A}\|_{s^{\star}} C_{\Lambda}|W|_{\infty} .
$$

\subsubsection{The bound $Z$}

This section is devoted to the construction of the bounds $\left\{Z_{k}(r)\right\}_{k=0, \ldots, M-1}$ satisfying

$$
\sup _{b_{1}, b_{2} \in B(r, s)}\left|\left[D T\left(\bar{x}+b_{1}\right) b_{2}\right]_{k}\right| \leq_{c w} Z_{k}(r), \quad k=0, \ldots, M-1,
$$

and of the tail bound $Z_{M}$ satisfying (25). To achieve this goal, it is convenient to factor the points $b_{1}, b_{2} \in B(r, s)$ as $b_{1}=r u, b_{2}=r v$ with $u, v \in B(1, s)$, to expand the derivative as a polynomial in $r$ and finally to compute a uniform bound for all $u, v \in B(1, s)$. Denote $u=\left[u_{0}, u_{1}, \ldots, u_{k}, \ldots\right]$, where each $u_{k}=\left(u_{k, 1}, u_{k, 2}\right) \in \operatorname{Mat}(n, \mathbb{R})^{2}$. For sake of simplicity of the presentation, both matrices $u_{k, 1}, u_{k, 2}$ are denoted as $u_{k}$. This abuse of notation can be used because what really matters in the estimates is the bound $\left|u_{k, 1}\right|,\left|u_{k, 2}\right| \leq_{c w} w_{k}^{-s}$ which is used to obtain the uniform estimates. We use a similar notation for $v_{k}$. 
Since $D T(\bar{x}+r u)=I-J D f(\bar{x}+r u)$, recalling the operator $J^{\dagger}$ defined in (19), we can consider the splitting

$$
\begin{aligned}
D T(\bar{x}+r u) r v & =[I-J D f(\bar{x}+r u)] r v \\
& =\left[I-J J^{\dagger}\right] r v-J\left[D f(\bar{x}+r u)-J^{\dagger}\right] r v
\end{aligned}
$$

so that

$$
\left|[D T(\bar{x}+r u) r v]_{k}\right| \leq_{c w}\left|\left[\left(I-J J^{\dagger}\right) r v\right]_{k}\right|+\left|\left[J\left(D f(\bar{x}+r u)-J^{\dagger}\right) r v\right]_{k}\right| .
$$

The construction of the bounds $\left\{Z_{k}(r)\right\}_{k=0, \ldots, M-1}$ follows as a combination of different intermediate vector bounds $Z^{0}, Z^{1}, Z^{2}$ satisfying

$$
\begin{aligned}
\left|\left[I-J J^{\dagger}\right] r v\right| & \leq_{c w} Z^{0} r, & & \forall v \in B(1, s), \\
\left|\left[D f(\bar{x}+r u)-J^{\dagger}\right] r v\right| & \leq_{c w} Z^{1} r+Z^{2} r^{2}, & & \forall u, v \in B(1, s),
\end{aligned}
$$

so that

$$
Z_{k}(r)=Z_{k}^{0} r+\left[\left|J_{M}\right|\left(Z^{1} r+Z^{2} r^{2}\right)^{M}\right]_{k}, \quad k=0, \ldots, M-1 .
$$

The uniform bound of the tail elements of $Z^{1}$ and $Z^{2}$ leads to the definition of $Z_{M}$. Let us now be more explicit about the bounds $Z^{0}, Z^{1}$ and $Z^{2}$.

The bound $Z^{0}$

By definition of $J$ and $J^{\dagger}$, the multiplication $\left[J J^{\dagger} x\right]_{k}=x_{k}$ for $k \geq M$. Thus the difference $I-J J^{\dagger}$ acts non trivially only on the finite dimensional projection $\Pi^{M} v$. Hence, considering the uniform bounds $\left|v_{k}\right| \leq_{c w} w_{k}^{-s}$, we can define $Z^{0}$ as

$$
\left(Z^{0}\right)_{k}= \begin{cases}{\left[\left|I_{2 n^{2} M}-J_{M} L_{M}\right|\left\{w_{j}^{-s} \mathbb{1}_{n}\right\}_{j=0}^{M-1}\right]_{k},} & k=0, \ldots, M-1 \\ 0, & k \geq M\end{cases}
$$

so that

$$
\left|\left[I-J J^{\dagger}\right] r v\right| \leq_{c w} Z^{0} r
$$

In the above formula $\left\{w_{j}^{-s} \mathbb{1}_{n}\right\}_{j=0}^{M-1}$ stands for the vector in $\mathbb{R}^{2 n^{2} M}$ that represents the vector of matrices $\left(\mathbb{1}_{n}, \mathbb{1}_{n}, w_{1}^{-s} \mathbb{1}_{n}, w_{1}^{-s} \mathbb{1}_{n}, \ldots, w_{M-1}^{-s} \mathbb{1}_{n}, w_{M-1}^{-s} \mathbb{1}_{n}\right)$ according with the representation introduced in Section 2.3.

Note that $J^{\dagger}$ is an almost inverse of $J$, indeed by definition $J_{M} L_{M} \approx I$. Therefore $\left|Z^{0}\right|$ is small and depends on the accuracy of the numerical method used to compute $J_{m}$.

The bounds $Z^{1}, Z^{2}$

Since the function $f(x)$ is quadratic in $x$, the derivative $D f(\bar{x}+r v)$ depends linearly on $r$, thus the idea is to express $\left(D f(\bar{x}+r u)-J^{\dagger}\right) r v$ as a quadratic polynomial in $r$. More precisely we aim at finding coefficients $c_{k, i}$ so that

$$
\left[\left(D f(\bar{x}+r u)-J^{\dagger}\right) r v\right]_{k}=\sum_{i=1,2} c_{k, i} r^{i}
$$

Then the construction of the components $Z_{k}^{i}$ can be derived as uniform bounds for $c_{k, i}$, $i=1,2$. To better understand the coefficients $c_{k, i}$, it is convenient to picture the operator $\left(D f(\bar{x}+r u)-J^{\dagger}\right) r v$ as an infinite dimensional matrix acting on the infinite dimensional vector $r v$. This is represented in Figure 1. On the top left corner, up to $k=m-1$, the matrix $J^{\dagger}$ is given by $D f^{(m)}(\bar{x})$ which implies that the only terms that survive the difference $D f^{(m)}(\bar{x}+r u)-D f^{(m)}(\bar{x})$ are the derivatives of the quadratic terms of $f_{j}$. For instance, 


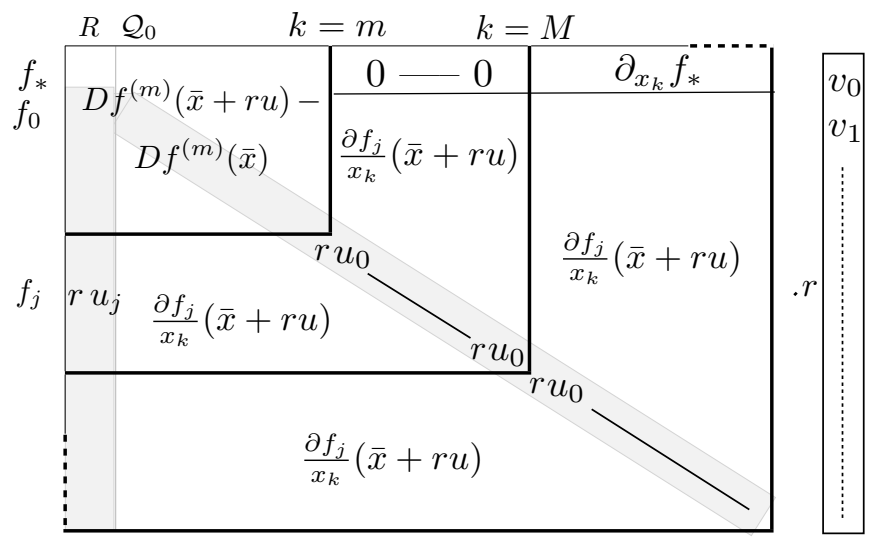

Figure 1: The operator $\left(D f(\bar{x}+r u)-J^{\dagger}\right) r v$ pictured as an infinite dimensional matrix acting on the infinite dimensional vector $r v$.

$\partial_{R} f_{0}(\bar{x}+r u)-\partial_{R} f_{0}(\bar{x})=\overline{\mathcal{Q}}_{0}+r u_{0}-\overline{\mathcal{Q}}_{0}=r u_{0}$. In the matrix of Figure 1 , the shadowed parts underline the positions corresponding to nonlinear terms of $f(x)$, namely $\partial_{R} f_{j}, \partial_{\mathcal{Q}_{j, 1}} f_{j}$ and $\partial_{\mathcal{Q}_{j, 2}} f_{j}$ for any $j \geq 0$. For $m \leq k<M$ the matrix $J^{\dagger}$ compensates the derivative of the phase condition and the derivative of the linear terms on the diagonal. However outside the diagonal, we have the terms $\partial_{x_{k}} f_{j}$ that are the derivatives of the convolution terms. Since the convolutions are linear in $\mathcal{Q}_{k, i}$, the derivatives depend only on the terms $\mathcal{A}_{k, i}$ and not on $(\bar{x}+r u)$. Finally, for $k \geq M$, we have the same situation as before with the adding of the derivatives of the phase condition, i.e. $\partial_{\mathcal{Q}_{k, 1}} f_{*}=2 I_{n^{2}}$.

Rewriting the convolution terms as

$$
\begin{aligned}
& (\mathcal{A} * \mathcal{Q})_{k, 1}=\sum_{k_{1}+k_{2}=k}\left(\mathcal{A}_{k_{1}, 1} \mathcal{Q}_{k_{2}, 1}-\mathcal{A}_{k_{1}, 2} \mathcal{Q}_{k_{2}, 2}\right), \\
& (\mathcal{A} * \mathcal{Q})_{k, 2}=\sum_{k_{1}+k_{2}=k}\left(\mathcal{A}_{k_{1}, 2} \mathcal{Q}_{k_{2}, 1}+\mathcal{A}_{k_{1}, 1} \mathcal{Q}_{k_{2}, 2}\right),
\end{aligned}
$$

recalling that $\mathcal{Q}_{k, 2}=-\mathcal{Q}_{-k, 2}$ and denoting by $s g(l)$ the sign of $l \in \mathbb{Z}$, one gets that

$$
\begin{aligned}
& c_{0,1}=\left[\begin{array}{c}
2 \sum_{k \geq M} v_{k}, \\
-\sum_{\substack{k_{1}+k_{2}=0 \\
\left|k_{2}\right| \geq m}}\left(\mathcal{A}_{k_{1}, 1}-s g\left(k_{2}\right) \mathcal{A}_{k_{1}, 2}\right) v_{\left|k_{2}\right|}
\end{array}\right], \quad c_{0,2}=\left[\begin{array}{c}
0 \\
u_{0} v_{0}+v_{0} u_{0}
\end{array}\right], \\
& c_{k, 1}=-\sum_{\substack{k_{1}+k_{2}=k \\
\left|k_{2}\right| \geq m}}\left[\begin{array}{l}
\left(\mathcal{A}_{k_{1}, 1}-s g\left(k_{2}\right) \mathcal{A}_{k_{1}, 2}\right) v_{\left|k_{2}\right|} \\
\left(\mathcal{A}_{k_{1}, 2}+s g\left(k_{2}\right) \mathcal{A}_{k_{1}, 1}\right) \\
v_{\left|k_{2}\right|}
\end{array}\right], \quad c_{k, 2}=\left[\begin{array}{c}
u_{k} v_{0}+v_{k} u_{0} \\
u_{k} v_{0}+v_{k} u_{0}
\end{array}\right] \text {, }
\end{aligned}
$$

for $k=1, \ldots, m-1$, and finally for $k \geq m$, one has that

$$
\left.c_{k, 1}=-\sum_{\substack{k_{1}+k_{2}=k \\
\left|k_{2}\right| \neq k}}\left[\begin{array}{l}
\left(\mathcal{A}_{k_{1}, 1}-s g\left(k_{2}\right) \mathcal{A}_{k_{1}, 2}\right. \\
\mathcal{A}_{k_{1}, 2}+s g\left(k_{2}\right) \mathcal{A}_{k_{1}, 1}
\end{array}\right) \begin{array}{l}
v_{\left|k_{2}\right|} \\
v_{\left|k_{2}\right|}
\end{array}\right], \quad c_{k, 2}=\left[\begin{array}{c}
u_{k} v_{0}+v_{k} u_{0} \\
u_{k} v_{0}+v_{k} u_{0}
\end{array}\right] .
$$


As mentioned above, the bounds $Z^{1}, Z^{2}$ need to be defined so that $Z_{k}^{1} \geq_{c w}\left|c_{k, 1}\right|$ and $Z_{k}^{2} \geq_{c w}\left|c_{k, 2}\right|$, for any $0 \leq k \leq M-1$. To achieve this, it is enough to substitute in the above expression the bounds $\left|u_{k}\right|,\left|v_{k}\right| \leq_{c w} w_{k}^{-s} \mathbb{1}_{n}$ and $\left| \pm \mathcal{A}_{j, 1} \pm \mathcal{A}_{j, 2}\right| \leq_{c w}\left|\mathcal{A}_{j, 1}\right|+\left|\mathcal{A}_{j, 2}\right|$. Since $\mathbb{1}_{n} \mathbb{1}_{n}=n \mathbb{1}_{n}$, one gets that

$$
\begin{aligned}
& \left|c_{0,2}\right| \leq_{c w} 2 n\left[\begin{array}{c}
0 \\
\mathbb{1}_{n}
\end{array}\right]=: Z_{0}^{2}, \\
& \left|c_{k, 2}\right| \leq_{c w} 2 n w_{k}^{-s}\left[\begin{array}{l}
\mathbb{1}_{n} \\
\mathbb{1}_{n}
\end{array}\right]=: Z_{k}^{2}, k \geq 1 .
\end{aligned}
$$

Consider now the coefficients $c_{k, 1}$. Due to the presence of series, the computation of the uniform bounds is a little more involved. The idea is to split the series into a finite part and an infinite tail part. The former is rigorously computed while the latter is estimated analytically. The sharpness of the bounds depends on the length of the finite part and on the decay rate of the coefficients $\mathcal{A}_{k}$. Splitting the series at $\left|k_{2}\right|=M$, we can write

$$
\begin{aligned}
& \left|c_{0,1}\right| \leq_{c w}\left[\sum_{\substack{k_{1}+k_{2}=0 \\
m \leq\left|k_{2}\right|<M}}\left(\left|\mathcal{A}_{k_{1}, 1}\right|+\left|\mathcal{A}_{k_{1}, 2}\right|\right) w_{k_{2}}^{-s} \mathbb{1}_{n}\right]+H_{0}=: Z_{0}^{1}, \\
& \left|c_{k, 1}\right| \leq_{c w} \sum_{\substack{k_{1}+k_{2}=k \\
m \leq\left|k_{2}\right|<M}}\left[\begin{array}{l}
\left(\left|\mathcal{A}_{k_{1}, 1}\right|+\left|\mathcal{A}_{k_{1}, 2}\right|\right) w_{k_{2}}^{-s} \mathbb{1}_{n} \\
\left.\left|\mathcal{A}_{k_{1}, 2}\right|+\left|\mathcal{A}_{k_{1}, 1}\right|\right) w_{k_{2}}^{-s} \mathbb{1}_{n}
\end{array}\right]+H_{k}=: Z_{k}^{1}, \quad k=1, \ldots, m-1 \\
& \left|c_{k, 1}\right| \leq \leq_{c w} \sum_{\substack{k_{1}+k_{2}=k \\
\left|k_{2}\right| \neq k,\left|k_{2}\right|<M}}\left[\left(\begin{array}{l}
\left(\left|\mathcal{A}_{k_{1}, 1}\right|+\left|\mathcal{A}_{k_{1}, 2}\right|\right) w_{k_{2}}^{-s} \mathbb{1}_{n} \\
\left(\left|\mathcal{A}_{k_{1}, 2}\right|+\left|\mathcal{A}_{k_{1}, 1}\right|\right) w_{k_{2}}^{-s} \mathbb{1}_{n}
\end{array}\right]+H_{k}=: Z_{k}^{1}, \quad k=m, \ldots, M-1,\right.
\end{aligned}
$$

where the matrices $H_{k}$ are the infinite tail part of the series and are defined as follows. For any $q>0, p>1$, define

$$
\zeta(q, p):=\frac{1}{(q)^{p}}+\frac{1}{(q+1)^{p}}+\frac{1}{p-1} \frac{1}{(q+1)^{p-1}},
$$

and

$$
H_{0}:=\left[\begin{array}{c}
2 \zeta(M, s) \mathbb{1}_{n} \\
h_{0} \mathbb{1}_{n}
\end{array}\right], \quad H_{k}:=h_{k}\left[\begin{array}{l}
\mathbb{1}_{n} \\
\mathbb{1}_{n}
\end{array}\right]
$$

where for $k \geq 0$

$$
h_{k}=\frac{\sqrt{2} n\|\mathcal{A}\|_{s^{\star}}}{(M-k)^{s^{\star}-s}}\left(\sum_{k_{2}=M-k}^{M-1} w_{k_{2}+k}^{-s} w_{k_{2}}^{-s}+2 \zeta(M, 2 s)\right) .
$$

Hence, one has the following result.

Lemma 2.7. Formula (39) holds for $H_{0}, H_{k}$ defined in (40).

Proof. First note that for any $M \geq 1$ and $s \geq 2$

$$
\sum_{k=M}^{\infty} \frac{1}{k^{s}}<\zeta(M, s)
$$


That can be seen from the fact that $\sum_{k=M}^{\infty} \frac{1}{k^{s}}=\frac{1}{(M)^{s}}+\frac{1}{(M+1)^{s}}+\sum_{k=M+2}^{\infty} \frac{1}{k^{s}}<\frac{1}{(M)^{s}}+$ $\frac{1}{(M+1)^{s}}+\int_{M+1}^{\infty} x^{-s} d x$. Hence, one has that

$$
\left|2 \sum_{k=M}^{\infty} w_{k}^{-s} \mathbb{1}_{n}\right| \leq_{c w} 2 \zeta(M, s) \mathbb{1}_{n} .
$$

That proves the result for the upper half of $H_{0}$. For the remaining terms, note that $\left(\left|\mathcal{A}_{k_{1}, 1}\right|+\right.$ $\left|\mathcal{A}_{k_{1}, 2}\right| \leq_{c w} \sqrt{2}\left|\mathcal{A}_{k_{1}}\right| \leq_{c w} \sqrt{2} \frac{\|\mathcal{A}\|_{s^{\star} \star}}{w_{k_{1}}^{\star \star}} \mathbb{1}_{n}$. Hence, for any $k \geq 0$, the tail part satisfies

$$
\begin{gathered}
\left|\sum_{\substack{k_{1}+k_{2}=k \\
\left|k_{2}\right| \geq M}}\left(\left|\mathcal{A}_{k_{1}, 1}\right|+\left|\mathcal{A}_{k_{1}, 2}\right|\right) w_{k_{2}}^{-s} \mathbb{1}_{n}\right| \leq_{c w} \sqrt{2}\|\mathcal{A}\|_{s^{\star}} \sum_{k_{2}=M}^{\infty}\left(\frac{1}{w_{k-k_{2}}^{s^{\star}}}+\frac{1}{w_{k+k_{2}}^{s^{\star}}}\right) \mathbb{1}_{n} w_{k_{2}}^{-s} \mathbb{1}_{n} \\
\leq_{c w} \frac{\sqrt{2} n\|\mathcal{A}\|_{s^{\star}}}{(M-k)^{s^{\star}-s}} \sum_{k_{2}=M}^{\infty}\left(\frac{1}{w_{k_{2}-k}^{s}}+\frac{1}{w_{k+k_{2}}^{s}}\right) w_{k_{2}}^{-s} \mathbb{1}_{n}
\end{gathered}
$$

where we used the fact that $s^{\star} \geq s$ and the relation $\mathbb{1}_{n} \mathbb{1}_{n}=n \mathbb{1}_{n}$. The result follows by applying (42) once the last series has be rewritten as

$$
\begin{aligned}
\sum_{k_{2}=M}^{\infty}\left(\frac{1}{w_{k_{2}-k}^{s}}+\frac{1}{w_{k+k_{2}}^{s}}\right) w_{k_{2}}^{-s} & =\sum_{k_{2}=M}^{\infty}\left(\frac{1}{w_{k+k_{2}}^{s}}\right) w_{k_{2}}^{-s}+\sum_{k_{2}=M-k}^{\infty}\left(\frac{1}{w_{k+k_{2}}^{s}}\right) w_{k_{2}}^{-s} \\
& \leq \sum_{k_{2}=M-k}^{\infty} w_{k+k_{2}}^{-s} w_{k_{2}}^{-s}+2 \sum_{k_{2}=M}^{\infty} w_{k_{2}}^{-2 s} .
\end{aligned}
$$

The bound $Z_{M}$

Recalling (25), $Z_{M}$ needs to satisfy $\frac{k^{s}}{M^{s}} \sup _{b_{1}, b_{2} \in B(r, s)}\left|\left[D T\left(\bar{x}+b_{1}\right) b_{2}\right]_{k}\right|_{\infty} \leq Z_{M}$ for any $k \geq M$. Since for $k \geq M$, the first term on the right hand side of (31) is zero, and we have that

$$
\begin{aligned}
\left|[D T(\bar{x}+r u) r v]_{k}\right|_{\infty} & \leq\left|\left[J\left(D f(\bar{x}+r u)-J^{\dagger}\right) r v\right]_{k}\right|_{\infty} \\
& \leq\left\|\Lambda_{k}^{-1}\right\|_{\infty}\left(\left|c_{k, 1}\right|_{\infty} r+\left|c_{k, 2}\right|_{\infty} r^{2}\right) .
\end{aligned}
$$

Thus it remains to find uniform bounds for $k \geq M$ of the coefficients $\left|c_{k, 1}\right|_{\infty},\left|c_{k, 2}\right|_{\infty}$ given in given in (34). To do this, we proceed as in the proof of Lemma 2.7, that is we bound each coefficient $\mathcal{A}_{k, i}$ in terms of the norm $\|\mathcal{A}\|_{s^{*}}$ and we estimate the remaining series using the following formula proved in [18]. For $k \geq M$, one has that

$$
\sum_{\substack{k_{1}+k_{2}=k \\\left|k_{1}\right| \neq k}} \frac{1}{w_{k_{1}}^{s} w_{k_{2}}^{s}} \leq \frac{1}{w_{k}^{s}}\left[1+2 \sum_{l=1}^{M} \frac{1}{l^{s}}+\frac{2}{M^{s-1}(s-1)}+\eta_{M}-\frac{1}{w_{2 k}^{s}}\right],
$$

where

$$
\eta_{M}=2\left[\frac{M}{M-1}\right]^{s}+\left[\frac{4 \log (M-2)}{M}+\frac{\pi^{2}-6}{3}\right]\left[\frac{2}{M}+\frac{1}{2}\right]^{s-2}
$$


Hence, letting $\delta:=1+2 \sum_{l=1}^{M} \frac{1}{l^{s}}+\frac{2}{M^{s-1}(s-1)}+\eta_{M}$, we get the bounds

$$
\begin{aligned}
\left|c_{k, 1}\right|_{\infty} & \leq \sqrt{2} n\|\mathcal{A}\|_{s^{\star}} \sum_{\substack{k_{1}+k_{2}=k \\
\left|k_{2}\right| \neq k}} \frac{1}{w_{k_{1}}^{s^{\star}} w_{k_{2}}^{s}} \leq \sqrt{2} n\|\mathcal{A}\|_{s^{\star}} \sum_{\begin{array}{c}
k_{1}+k_{2}=k \\
\left|k_{2}\right| \neq k
\end{array}} \frac{1}{w_{k_{1}}^{s} w_{k_{2}}^{s}} \leq \frac{\sqrt{2} n\|\mathcal{A}\|_{s^{\star}}}{w_{k}^{s}} \delta, \\
\left|c_{k, 2}\right|_{\infty} & \leq \frac{2 n}{w_{k}^{s}}
\end{aligned}
$$

Finally, combining (15) with $k \geq M$, it follows that $\left\|\Lambda_{k}^{-1}\right\|_{\infty} \leq \frac{C_{\Lambda}}{M}$, and then we let

$$
Z_{M}:=\frac{C_{\Lambda}}{M^{s+1}}\left(\sqrt{2}\|\mathcal{A}\|_{s^{\star}} \delta r+2 n r^{2}\right) .
$$

Remark 2.8. The quantities $Y_{M}, h_{k}$ and $Z_{M}$ defined respectively in (29), (41) and (45) depend on $\|\mathcal{A}\|_{s^{\star}}<\infty$. However it is easy to see that formulas (29) and (41) can be rewritten respectively with the quantities $\sup _{j \geq M-m}\left\{\left|\mathcal{A}_{j}\right|_{\infty} w_{j}^{s^{\star}}\right\}$ and $\sup _{|j| \geq M-k}\left\{\left|\mathcal{A}_{j}\right|_{\infty} w_{j}^{s^{*}}\right\}$ instead of $\|\mathcal{A}\|_{s^{*}}$. Therefore, a more detailed knowledge of the behavior of the coefficients $\mathcal{A}_{k}$ should help choosing the computational parameter $M$ in order to improve the estimates. For instance, if there exists $\varepsilon$ such that $\left|\mathcal{A}_{k}\right|_{\infty} w_{k}^{s^{\star}}<\varepsilon$ for any $|k|>m_{\mathcal{A}}$, then setting $M>m+m_{\mathcal{A}}$, the estimate (29) holds with $\varepsilon$ in place of $\|\mathcal{A}\|_{s^{*}}$.

\section{Computing stable and unstable bundles of periodic orbits via Floquet normal forms}

Consider an autonomous differential equation

$$
\dot{y}=g(y), \quad g \in C^{1}\left(\mathbb{R}^{n}\right)
$$

and suppose that $\gamma(t)$ is a $\tau$-periodic solution with $\gamma(0)=\gamma_{0}$. Denote by $\Gamma=\{\gamma(t), t \in$ $[0, \tau]\}$ the support of $\gamma$ and for any $\theta \in[0, \tau]$, define $\gamma_{\theta}(t)=\gamma(t+\theta)$ the phase-shift reparametrization of $\Gamma$. Being autonomous, system (46) has the property that any of the curves $\gamma_{\theta}(t)$ is a $\tau$-periodic solution satisfying $\gamma_{\theta}(0)=\gamma(\theta)$. We refer to $\Gamma$ as the periodic orbit and $\gamma_{\theta}$ as the periodic solutions.

Definition 2 (Monodromy matrix). Let $\gamma: \mathbb{R} \rightarrow \mathbb{R}^{n}$ be a $\tau$-periodic solution (46) and let $\Phi_{\theta}(t)$ be the unique solution of the non-autonomous linear problem

$$
\left\{\begin{array}{l}
\dot{\Phi}_{\theta}=\nabla g\left(\gamma_{\theta}(t)\right) \Phi_{\theta} \\
\Phi_{\theta}(0)=I_{n}
\end{array}\right.
$$

The matrix $\Phi_{\theta}(\tau)$ is called the monodromy matrix of $\gamma_{\theta}(t)$.

Having chosen $\gamma(t)=\gamma_{0}(t)$, in the following we identify $\Phi(\tau)=\Phi_{0}(\tau)$. The next two results are classical results and they are direct consequences of $\Phi_{\theta}(t)$ being a fundamental matrix solution. For sake of completeness, we present their proofs.

Lemma 3.1. For any $\theta \in[0, \tau]$, the solution $\Phi_{\theta}(t)$ of (46) satisfies

$$
\Phi_{\theta}(n \tau+t)=\Phi_{\theta}(t) \Phi_{\theta}(\tau)^{n}, \forall t \in \mathbb{R}, \forall n \in \mathbb{N}
$$


Proof. Without loss of generality, let us consider $\theta=0$. We proceed by induction on $n \geq 0$. For $n=0$ the result is obvious. Suppose the result holds for $n-1$. Then

$$
\Phi(n \tau)=\Phi((n-1) \tau+\tau)=\Phi(\tau) \Phi(\tau)^{n-1}=\Phi(\tau)^{n} .
$$

Define

$$
\Psi(t)=\Phi(t+n \tau) \Phi(n \tau)^{-1} .
$$

It follows that $\Psi(0)=I_{n}$ and that

$$
\dot{\Psi}(t)=\dot{\Phi}(n \tau+t) \Phi(n \tau)^{-1}=A(n \tau+t) \Phi(n \tau+t) \Phi(n \tau)^{-1}=A(t) \Psi(t) .
$$

From unicity of solutions of the initial value problem, one has that $\Psi(t)=\Phi(t)$. Hence,

$$
\Phi(t+n \tau)=\Phi(t) \Phi(n \tau)=\Phi(t) \Phi(\tau)^{n}, \forall t \in \mathbb{R} .
$$

Lemma 3.2. The matrices $\Phi_{\theta}(\tau)$ are equivalent under conjugation. In particular

$$
\Phi_{\theta}(\tau)=\Phi(\theta) \Phi(\tau) \Phi(\theta)^{-1} .
$$

Proof. The matrix $\tilde{\Phi}(t):=\Phi(t+\theta)$ is a solution of the equation $\dot{y}=\nabla g(\gamma(t+\theta)) y=$ $\nabla g\left(\gamma_{\theta}(t)\right)$, with $\tilde{\Phi}(0)=\Phi(\theta)$. Since $\Phi_{\theta}(t)$ is the principal fundamental solution,

$$
\tilde{\Phi}(t)=\Phi_{\theta}(t) \Phi(\theta) .
$$

It follows

$$
\Phi_{\theta}(t)=\tilde{\Phi}(t) \Phi(\theta)^{-1}=\Phi(t+\theta) \Phi(\theta)^{-1}, \quad \forall t .
$$

Using Lemma 3.1, one can then conclude that

$$
\Phi_{\theta}(\tau)=\Phi(\tau+\theta) \Phi(\theta)^{-1}=\Phi(\theta) \Phi(\tau) \Phi(\theta)^{-1} .
$$

The previous result implies that all monodromy matrices $\Phi_{\theta}(\tau)$ have the same eigenvalues. That motivates the following definition.

Definition 3. The eigenvalues $\sigma_{j}$ of the monodromy matrix $\Phi(\tau)$ are called the Floquet multipliers of the periodic orbit $\Gamma$.

As already mentioned in Section 1, in the theory of dynamical systems the monodromy matrix $\Phi(\tau)$ associated to a periodic solution $\gamma(t)$ plays a fundamental role since it contains information about the linear stability of $\gamma$. Indeed, as shown in Proposition 2.122 in [2], the Floquet multipliers of $\gamma(t)$ are in fact the eigenvalues of $D \mathbf{P}(\gamma(0))$, where $\mathbf{P}(x)$ denotes the Poincaré map of $\gamma(t)$ on a $(n-1)$-dimensional hypersurface transversal to $\gamma$ at $\gamma(0)$. Moreover, at least one of the Floquet multipliers $\sigma_{j}$ of $\Phi(\tau)$ equals one, corresponding to the eigenvector $\dot{\gamma}(0)$. Hence, we denote by $\sigma_{n}=1$ the Floquet multiplier corresponding to $\dot{\gamma}(0)$ and by $\left\{\sigma_{j}\right\}_{j=1, \ldots, n-1}$ the set of nontrivial Floquet multipliers. We refer to Section 2.4 in [2] for a more extensive analysis of the links between Poincaré sections and Floquet theory. Based on the above discussion, let us introduce the notion of stability of a periodic orbit.

Definition 4. Let $\Gamma=\{\gamma(t), t \in[0, \tau]\}$ be a $\tau$-periodic orbit of the system (46) and let $\left\{\sigma_{j}\right\}_{j=1, \ldots, n-1}$ be the corresponding set of nontrivial Floquet multipliers. We say that 
- $\Gamma$ is stable if $\forall j \in\{1, \ldots, n-1\},\left|\sigma_{j}\right|<1$;

- $\Gamma$ is unstable if $\exists j \in\{1, \ldots, n-1\}$ such that $\left|\sigma_{j}\right|>1$.

Moreover, if $p<n-1$ Floquet multipliers have modulus less than one, and $q<n-p$ Floquet multipliers have modulus greater than one, $\Gamma$ is said to have $p$ stable directions and $q$ unstable directions.

Let us mention that there is a variant to the Floquet normal form introduce in Theorem 1.1, namely there exist a constant (possibly complex) matrix $B$ and a nonsingular (possibly complex) continuously differentiable, $\tau$-periodic matrix function $P(t)$ such that $\Phi(t)=P(t) e^{B t}$. We refer to Theorem 2.83 in [2] for more details and for the proof. Therefore, there exists a (possibly complex) matrix B such that $\Phi(\tau)=e^{B \tau}$. Denoting by $\lambda_{j}$ the eigenvalues of $B$, it follows that $\sigma_{j}=e^{\tau \lambda_{j}}$ is a Floquet multiplier. Note that for a given $\sigma_{j}$, the solution $\lambda_{j}$ of $\sigma_{j}=e^{\tau \lambda_{j}}$ is not uniquely defined. Indeed for any $k \in \mathbb{Z}, e^{\tau\left(\lambda_{j}+i \frac{2 k \pi}{\tau}\right)}=\sigma_{j}$. This reflects the fact that in the complex Floquet normal form $\Phi(t)=P(t) e^{B t}$, the matrix $B$ is also not uniquely defined. In the literature it is common to call a Floquet exponent associated to $\sigma_{j}$ any complex number $\lambda_{j}$ so that $\sigma_{j}=e^{\tau \lambda_{j}}$. On the converse, for any $\sigma_{j}$ there is a unique real number $l_{j}$ so that $\left|\sigma_{j}\right|=e^{l_{j} \tau}$. That motivates the following definition.

Definition 5. A Lyapunov exponent associated to a Floquet multiplier $\sigma_{j}$ is the unique real number $l_{j}$ so that $\left|\sigma_{j}\right|=e^{l_{j} \tau}$.

Note that using the notion Lyapunov exponents, a definition of stability of a periodic orbit similar to the one of Definition 4 can be introduced. Indeed, given a $\tau$-periodic orbit $\Gamma=\{\gamma(t), t \in[0, \tau]\}$ of (46) and considering $\left\{l_{j}\right\}_{j=1, \ldots, n-1}$ to be the corresponding set of nontrivial Lyapunov exponents, we say that $\Gamma$ is stable if $l_{j}<0, \forall j=1, \ldots, n-1$ and that $\Gamma$ is unstable if there exists $j \in\{1, \ldots, n-1\}$ such that $l_{j}>0$.

Given a real $n \times n$ diagonalizable matrix $A$, let us introduce the notation $\Sigma(A)=$ $\left\{\alpha_{k}, v_{k}\right\}_{k=1, \ldots, n}$ to denote the eigendecomposition of the square matrix $A$, i.e. $A v_{k}=\alpha_{k} v_{k}$, for all $k=1, \ldots, n$.

The following result shows how the information from the couple $(R, Q(t))$ coming from the Floquet normal form $\Phi(t)=Q(t) e^{R t}$ can directly be used to study the dynamical properties of the periodic orbit $\Gamma$. More explicitly, it demonstrates that the stability of $\Gamma$ can be determined by the eigenvalues of $R$ while the stable and unstable tangent bundles of $\Gamma$ can be retrieved from the action of $Q(t)$ (with $t \in[0, \tau]$ ) on the eigenvectors of $R$.

Theorem 3.3. Assume that $\Gamma=\{\gamma(t), t \in[0, \tau]\}$ is a $\tau$-periodic orbit of (46) and consider $\Phi(t)$ the fundamental matrix solution of the non-autonomous linear equation $\dot{y}=\nabla g(\gamma(t)) y$ such that $\Phi(0)=I$. Suppose that a Floquet normal form decomposition of Theorem 1.1) $\Phi(t)=Q(t) e^{R t}$ is known. Assume that the real $n \times n$ matrix $R$ is diagonalizable and let $\Sigma(R)=\left\{\mu_{j}, v_{j}\right\}_{j=1, \ldots, n}$ the eigendecomposition of $R$. Then the Lyapunov exponents $l_{j}$ of $\Gamma$ are given by

$$
l_{j}=\operatorname{Re}\left(\mu_{j}\right) .
$$

Furthermore, for any $\theta \in[0, \tau]$, if one defines

$$
w_{j}^{\theta}:=Q(\theta) v_{j},
$$

then $w_{j}^{\theta}$ is an eigenvector of $\Phi_{\theta}(\tau)$ associated to the Lyapunov exponent $l_{j}$. Note that $w_{j}^{\theta}$ is a smooth $2 \tau$-periodic function of $\theta$. 
Proof. Consider the eigendecomposition $\Sigma(R)=\left\{\mu_{j}, v_{j}\right\}_{j=1, \ldots, n}$ of the diagonalizable matrix $R$, meaning that the set $\left\{v_{1}, \ldots, v_{n}\right\}$ consists of $n$ linearly independent eigenvectors of $R$. By Lemma 3.1, one has that $\Phi(\tau)^{2}=\Phi(2 \tau)$. Since $Q(t)$ is $2 \tau$-periodic and $Q(0)=I$, it follows that $\Phi(2 \tau)=e^{R 2 \tau}$. Since $R$ is diagonalizable, $\Phi(2 \tau)=e^{R 2 \tau}$ is also diagonalizable. Since $\Phi(2 \tau)=\Phi(\tau)^{2}$ and since the matrix $\Phi(\tau)$ is invertible and defined over the field of complex number (which has zero characteristic), then it can then be showed that $\Phi(\tau)$ is also diagonalizable. Now, since $\Phi(2 \tau)=\Phi(\tau)^{2}$ one has that if $(\sigma, w) \in \Sigma(\Phi(\tau))$, then $\left(\sigma^{2}, w\right) \in \Sigma(\Phi(2 \tau))$. Combining this last point with $\Phi(\tau), \Phi(2 \tau)$ being diagonalizable implies that the eigenspaces of $\Phi(\tau)$ and $\Phi(2 \tau)$ are in one-to-one correspondence. That implies the existence of a set $\left\{\sigma_{j}\right\}_{j=1, \ldots, n}$ such that $\Sigma(\Phi(\tau))=\left\{\sigma_{j}, v_{j}\right\}_{j=1, \ldots, n}$. From the property of the exponential matrix operator, $\Sigma(\Phi(2 \tau))=\left\{e^{\mu_{j} 2 \tau}, v_{j}\right\}_{j=1, \ldots, n}=\Sigma\left(\Phi(\tau)^{2}\right)=\left\{\sigma_{j}^{2}, v_{j}\right\}_{j=1, \ldots, n}$. This implies that $\sigma_{j}^{2}=e^{\mu_{j} 2 \tau}$ for any $j=1, \ldots, n$. Note that $l_{j}=\operatorname{Re}\left(\mu_{j}\right)$ is the unique real number so that $\left|\sigma_{j}\right|=e^{l_{j} \tau}$. Hence, $l_{j}$ is a Lyapunov exponent associated to the Floquet multipliers $\sigma_{j}$.

Now, from (49), one has that

$$
\Phi_{\theta}(2 \tau)=\Phi(2 \tau+\theta) \Phi(\theta)^{-1}=Q(\theta) e^{(2 \tau+\theta) R} e^{-R \theta} Q(\theta)^{-1}, \quad \forall \theta \in[0, \tau]
$$

thus

$$
\Phi_{\theta}(2 \tau) Q(\theta) v_{j}=Q(\theta) e^{2 \tau R} v_{j}=e^{2 \tau \mu_{j}} Q(\theta) v_{j}
$$

showing that $\Sigma\left(\Phi_{\theta}(2 \tau)\right)=\left\{e^{2 \tau \mu_{j}}, Q(\theta) v_{j}\right\}$. Applying the same argument than above, one can conclude that $\Sigma\left(\Phi_{\theta}(\tau)\right)=\left\{\sigma_{j}, Q(\theta) v_{j}\right\}_{j=1, \ldots, n}$ forms an eigendecomposition of the matrix $\Phi_{\theta}(\tau)$. Hence, $w_{j}^{\theta}=Q(\theta) v_{j}$ is an eigenvector of $\Phi_{\theta}(\tau)$. By the smoothness and the $2 \tau$-periodicity of the matrix function $Q(\theta)$, one can conclude that $w_{j}^{\theta}=Q(\theta) v_{j}$ is also a smooth $2 \tau$-periodic function of $\theta$.

Recall (51) and consider $w_{j}^{\theta}=a_{j}^{\theta}+i b_{j}^{\theta}$. We define the stable and unstable subspaces $E_{s}^{\theta}, E_{u}^{\theta} \subset T_{\gamma(\theta)} \mathbb{R}^{n}$ of the periodic orbit $\Gamma$ at the point $\gamma(\theta)$ as

$$
\begin{aligned}
& E_{s}^{\theta}=\operatorname{Span}\left\{a_{i}^{\theta}, b_{i}^{\theta}:\left|\sigma_{j}\right|<1\right\} \\
& E_{u}^{\theta}=\operatorname{Span}\left\{a_{i}^{\theta}, b_{i}^{\theta}:\left|\sigma_{j}\right|>1\right\} .
\end{aligned}
$$

That allows us to define the following.

Definition 6. We define the stable and unstable tangent bundles of $\Gamma$ respectively by

$$
\begin{gathered}
E_{s}, E_{u} \subset T_{\Gamma} \mathbb{R}^{n} \\
E_{s}=\bigcup_{\theta \in[0, \tau]}\{\gamma(\theta)\} \times E_{s}^{\theta}, \quad E_{u}=\bigcup_{\theta \in[0, \tau]}\{\gamma(\theta)\} \times E_{u}^{\theta} .
\end{gathered}
$$

It is important to remark that from the conclusion of Theorem 3.3, the complete structure of the stable and unstable bundles can be recovered by the action of the matrix function $Q(t)$ on the eigenvectors of $R$, which themselves correspond to the stable and unstable directions at the point $\gamma(0)$ on $\Gamma$. Also, the proof of Theorem 3.3 is constructive in the sense that combined with the rigorous computational method of Section 2, it provides a computationally efficient direct way to obtain the eigenvectors $w_{j}^{\theta}$ of $\Phi_{\theta}(\tau)$, which are the ingredients defining the bundles of Definition 6. Note that one could be tempted to use the fact that $\Phi(\tau)=Q(\tau) e^{R \tau}$ and then attempt to compute the eigendecomposition of $\Phi(\tau)$ directly. However, that would imply having to compute the exponential of an interval 
valued matrix, which turns out to be a difficult task (e.g. see [22], [23]). This being said, the rigorous computation of the eigendecomposition of the interval matrix $R$ is not completely straightforward. We addressed this problem by adapting the computational method based on the radii polynomials in order to enclose all the solution $\left\{\mu_{k}, v_{k}\right\}$ of the nonlinear problem $(R-\mu I) v=0$ with some scaling constrain to isolate the solution. Further details on the enclosure of the eigendecomposition of interval matrices is presented in [24].

\section{Applications}

In this section, we present some applications, where we construct rigorously tangent stable and unstable bundles of some periodic orbits of the Lorenz equations in Section 4.1 and of the $\zeta^{3}$-model in Section 4.2. Finally, in Section 4.3, we discuss how to recover a posteriori the Floquet multipliers associated to the periodic orbit $\gamma(t)$, that is the eigenvalues of the matrices $\Phi_{\theta}(\tau)$. Note that all rigorous computations were performed in Matlab with the interval arithmetic package Intlab [25], while the codes necessary to compute the Floquet normal forms in the context of the Lorenz equations can be found in [29].

\subsection{Bundles of periodic orbits in the Lorenz equations}

Consider the following three dimensional system of ODEs, known as the Lorenz equations

$$
\left\{\begin{array}{l}
\dot{u}_{1}=\sigma\left(u_{2}-u_{1}\right) \\
\dot{u}_{2}=\rho u_{1}-u_{2}-u_{1} u_{3} \\
\dot{u}_{3}=u_{1} u_{2}-\beta u_{3}
\end{array}\right.
$$

with the classical choice of parameters $\beta=8 / 3, \sigma=10$ and $\rho$ left as a bifurcation parameter. First we rigorously compute a family of periodic solutions $\gamma(t)=\left[\gamma^{1}, \gamma^{2}, \gamma^{3}\right](t)$ of (52) in the form

$$
\gamma^{j}(t)=\sum_{k \in \mathbb{Z}} \xi_{k}^{j} e^{i k \frac{2 \pi}{\tau_{\gamma}} t}, \quad j=1,2,3
$$

one of each in a ball of radius $r_{\gamma}$, with respect to the $\Omega^{s^{*}}$ norm, around a numerical solution $\left[\bar{\tau}_{\gamma}, \bar{\xi}_{k}\right], \bar{\xi}_{k}=0$ for $|k|>m_{\gamma}$. More explicitly, for a choice of the parameter $\rho$ and a decay rate $s^{\star} \geq 2$, we prove the existence of a $\tau_{\gamma}$-periodic solution $\gamma(t)$ so that

$$
\begin{gathered}
\left|\tau_{\gamma}-\bar{\tau}_{\gamma}\right| \leq r_{\gamma} \\
\left|\operatorname{Re}\left(\xi_{k}\right)-\operatorname{Re}\left(\bar{\xi}_{k}\right)\right|_{\infty} \leq r_{\gamma} w_{k}^{-s^{\star}}, \quad\left|\operatorname{Im}\left(\xi_{k}\right)-\operatorname{Im}\left(\bar{\xi}_{k}\right)\right|_{\infty} \leq r_{\gamma} w_{k}^{-s^{\star}} .
\end{gathered}
$$

Note that $\xi_{k} \in \mathbb{C}^{3}$ and $\xi_{-k}=\mathcal{C}\left(\xi_{k}\right)$, where $\mathcal{C}(z)$ is the complex conjugate of $z$. The existence of such solutions has been achieved by applying a modified version of the method discussed in the previous section. Even with some technical differences, the philosophy is the same. Rewrite the system of ODEs as a infinite dimensional algebraic system where $\tau_{\gamma}$ and the Fourier coefficients $\xi_{k}$ are the unknowns, then consider a finite dimensional projection and compute a numerical approximate solution $\bar{\tau}_{\gamma},\left(\bar{\xi}_{k}\right)_{k}$. Then, using the radii polynomials, prove the existence, in a suitable Banach space, of a genuine solution $\tau_{\gamma},\left(\xi_{k}\right)_{k}$ of the infinite dimensional problem in a small ball containing the approximate solution. In the next subsection some of the results are presented.

Then, combining the method discussed in Section 2 and Theorem 3.3, we rigorously enclose the stable and unstable tangent bundles of some of the rigorously computed periodic 
orbits $\gamma(t)$. This first requires the computation of the Floquet normal form of the principal fundamental matrix solution $\Phi(t)$ of the linearized system along $\gamma(t)$. More explicitly $\Phi(t)$ is the solution for $t \in\left[0, \tau_{\gamma}\right]$ of the non-autonomous system

$$
\left\{\begin{array}{l}
\dot{\Phi}=\nabla g(\gamma(t)) \Phi \\
\Phi(0)=I_{n}
\end{array}\right.
$$

where $g$ is the right hand side of (52), $\nabla g$ denotes the Jacobian of the right hand side of system (52) and $I$ is the $3 \times 3$ identity matrix. The former system is nothing more than a particular case of (1), where $A(t)=\nabla g(\gamma(t))$ and $n=3$. We now apply the computational method presented in Section 2 to compute the Floquet normal form of $\Phi(t)$, which is the solution of (55). In particular a constant matrix $R$ and the Fourier coefficients $\mathcal{Q}_{k}$ of a $2 \tau_{\gamma}$-periodic function $Q(t)$ is computed, so that $\Phi(t)=Q(t) e^{R t}$ solves (55). Once the computation of $R$ and the $\mathcal{Q}_{k}$ is done, following the conclusion of Theorem 3.3, we compute $\Sigma(R)=\left\{\left(\mu_{j}, v_{j}\right) \mid j=1, \ldots, n\right\}$, we derive from the Lyapunov exponents $l_{j}:=\operatorname{Re}\left(\mu_{j}\right)$ the linear stability of the periodic orbit $\Gamma$ and using the eigenvectors $\left\{v_{1}, \ldots, v_{n}\right\}$ of $R$ we construct the tangent bundles as defined in Definition 6 and given by the formula (51).

Computation of $R$ and $Q_{k}$

To begin with, let us explicitly write the Jacobian of (52)

$$
\nabla g(u)=\left[\begin{array}{ccc}
-\sigma & \sigma & 0 \\
\rho-u_{3} & -1 & -u_{1} \\
u_{2} & u_{1} & -\beta
\end{array}\right]
$$

and, recalling (5), the Fourier coefficients $\mathcal{A}_{k}$ of $A(t)=\nabla g(\gamma(t))$

$$
\mathcal{A}_{0}=\left[\begin{array}{ccc}
-\sigma & \sigma & 0 \\
\rho-\xi_{0}^{3} & -1 & -\xi_{0}^{1} \\
\xi_{0}^{2} & \xi_{0}^{1} & -\beta
\end{array}\right], \quad \mathcal{A}_{k}=\left[\begin{array}{ccc}
0 & 0 & 0 \\
-\xi_{k}^{3} & 0 & -\xi_{k}^{1} \\
\xi_{k}^{2} & \xi_{k}^{1} & 0
\end{array}\right], \quad k \geq 1 .
$$

Note that the hypothesis (54) for $\xi_{k}$ to lie in a ball centered at $\bar{\xi}_{k}$ implies that $\|\mathcal{A}\|_{s^{\star}}<\infty$. The computation of the approximate solution $\bar{R}, \overline{\mathcal{Q}}_{k, 1}, \overline{\mathcal{Q}}_{k, 2}$ has been done as follow: consider the approximation $\bar{\gamma}(t)=\sum_{|k| \leq m_{\gamma}} \bar{\xi}_{k} e^{i k 2 \pi t / \bar{\tau}_{\gamma}}$ of the periodic orbit $\gamma(t)$ and numerically solve system (55) up to time $2 \bar{\tau}_{\gamma}$. Denote by $\bar{y}\left(2 \bar{\tau}_{\gamma}\right)$ the obtained result and numerically compute

$$
\mathscr{R}=\log \left(\bar{y}\left(2 \bar{\tau}_{\gamma}\right)\right) .
$$

Neglect the imaginary part of $\mathscr{R}$ and consider only its real part. Then numerically integrate the system (3) up to time $2 \bar{\tau}_{\gamma}$ with $\mathscr{R}$ in place of $R$ yielding the solution $\mathscr{Q}(t)$. Fix the finite dimensional parameter $m$ and compute from $\mathscr{Q}(t)$ the matrices $\mathscr{Q}_{k, 1}, \mathscr{Q}_{k, 2}$, respectively the real and imaginary part of the Fourier coefficients with $|k|<m$. Finally the vector $\left(\mathscr{R},\left(\mathscr{Q}_{k, 1}, \mathscr{Q}_{k, 2}\right)_{k}\right)$ is considered as starting point for a Newton iteration scheme applied on the finite dimensional projection defined by (11). Denote the output of the iterative process by $\bar{x}=\left(\bar{R},\left(\overline{\mathcal{Q}}_{k, 1}, \overline{\mathcal{Q}}_{k, 2}\right)_{k}\right)$, that is an approximated solution $f^{(m)}(\bar{x}) \approx 0$ up to a desired accuracy, with $f^{(m)}$ defined in (11).

Consider $\Lambda_{k}$ given by (16). Note that in the case of the three-dimensional vector field (52), $\Lambda_{k}$ is a $18 \times 18$ matrix and one could compute its inverse analytically using the mathematical software Maple. After having computed $\Lambda_{k}^{-1}$ one needs to check that the chosen $m$ satisfies $m>K$ where $K$ is the same as in Lemma 2.2, otherwise increase $m$.

Then the computational parameter $M>m$ has to be fixed: in the following, as well as in all the presented computations, we assume

$$
M>m+m_{\gamma} .
$$


Given $M$, we compute the constant $C_{\Lambda}(M)$ introduced in Lemma 2.2. As already mentioned in Section 2.3, the knowledge of the behaviour of the matrices $\mathcal{A}_{2 k}$ for $k \geq M$ allows computing a sharper bound $C_{\Lambda}$ than the one given in Section 6.1 in the general setting. Indeed, since for any $k>m_{\gamma}$, we have $\left|\operatorname{Re}\left(\xi_{k}\right)\right|_{\infty} \leq r_{\gamma} w_{k}^{s^{*}}$ and $\left|\operatorname{Im}\left(\xi_{k}\right)\right|_{\infty} \leq r_{\gamma} w_{k}^{s^{*}}$, it follows that $\left|\mathcal{A}_{2 k, 1}\right|,\left|\mathcal{A}_{2 k, 2}\right| \leq \mathbb{A}_{M}$, for all $k \geq M$, where

$$
\mathbb{A}_{M}:=\frac{r_{\gamma}}{2 M^{s^{*}}}\left[\begin{array}{lll}
0 & 0 & 0 \\
1 & 0 & 1 \\
1 & 1 & 0
\end{array}\right]
$$

Thus we can replace the matrix $\bar{\Lambda}_{M}$ given in (66) by

$$
\bar{\Lambda}_{M}:=\left[\begin{array}{cc}
{\left[\mathbb{A}_{M}\right]_{n}} & \left|R^{*}-\left[\mathcal{A}_{0}\right]_{n}\right|+\left[\mathbb{A}_{M}\right]_{n} \\
\left|R^{*}-\left[\mathcal{A}_{0}\right]_{n}\right|+\left[\mathbb{A}_{M}\right]_{n} & {\left[\mathbb{A}_{M}\right]_{n}}
\end{array}\right] .
$$

Moreover, note that $\mathbb{A}_{M}$ has null diagonal, therefore we define $C_{\Lambda}$ as

$$
C_{\Lambda}:=\frac{1}{\min _{i}\left\{\frac{2 \pi}{2 \tau}-\frac{1}{M} \sum_{j \neq i} \bar{\Lambda}_{M}(i, j)\right\}}
$$

Notice the difference with the general formula of $C_{\Lambda}$ given in (67). Now that the parameters $m, M$ are chosen and $C_{\Lambda}$ is computed, one can compute, for a given choice of decay rate $s \in\left[2, s^{\star}\right]$, the coefficients $Y_{k}, Z_{k}, k=0, \ldots, M$ as shown in Section 2.4.

As already mentioned in Remark 2.8 , the bound $\|\mathcal{A}\|_{s^{\star}}<\infty$ is sufficient to proceed with the computational method, we want to deepen what was highlighted in : it is easy to see that

$$
\left|\mathcal{A}_{k}\right|_{\infty} \leq \sqrt{2} r_{\gamma} \frac{1}{w_{k}^{s^{\star}}}, \quad \forall k>m_{\gamma}
$$

thus the above choice of $M$ implies that the tail elements $H_{0}, H_{k}$ in (39) only contain the terms $\mathcal{A}_{j}$ 's satisfying $\left|\mathcal{A}_{j}\right|_{\infty} \leq \sqrt{2} r_{\gamma} w_{j}^{-s^{\star}}$. Therefore the subsequent estimate for $h_{k}$ can be improved by replacing $\|\mathcal{A}\|_{s^{\star}}$ with $\sqrt{2} r_{\gamma}$, giving

$$
h_{k}=\frac{2 n r_{\gamma}}{(M-k)^{s^{\star}-s}}\left(\sum_{k_{2}=M-k}^{M-1} w_{k_{2}+k}^{-s} w_{k_{2}}^{-s}+2 \zeta(M, 2 s)\right), \quad \text { for } k=1, \ldots, m-1 .
$$

On the other hand, for $k=m, \ldots, M-1$ the above estimate does not holds, since $M$ could be less then $k+m_{\gamma}$. Therefore, for those $k$ such that $M>k+m_{\gamma}$ we continue using the previous formula for $h_{k}$ while for those $k$ such that $M \leq k+m_{\gamma}$ we proceed as follow. Rewrite explicitly (part of) the vector $H_{k}$

$$
\begin{aligned}
H_{k} & =\sum_{\substack{k_{1}+k_{2}=k \\
M \leq\left|k_{2}\right| \leq k+m_{\gamma}}}\left(\left|\mathcal{A}_{k_{1}, 1}\right|+\left|\mathcal{A}_{k_{1}, 2}\right|\right) w_{k_{2}}^{-s} \mathbb{1}_{n}+2 r_{\gamma} \sum_{\substack{k_{1}+k_{2}=k \\
\left|k_{2}\right|>k+m_{\gamma}}} w_{k_{1}}^{-s^{\star}} \mathbb{1}_{n} w_{k_{2}}^{-s} \mathbb{1}_{n} \\
& =\sum_{\substack{k_{1}+k_{2}=k \\
M \leq\left|k_{2}\right| \leq k+m_{\gamma}}}\left(\left|\mathcal{A}_{k_{1}, 1}\right|+\left|\mathcal{A}_{k_{1}, 2}\right|\right) w_{k_{2}}^{-s} \mathbb{1}_{n}+2 n r_{\gamma} \sum_{\substack{k_{1}+k_{2}=k \\
\left|k_{2}\right|>k+m_{\gamma}}}^{-s^{\star}} w_{k_{2}}^{-s} \mathbb{1}_{n} .
\end{aligned}
$$

The first is a finite sum and it is rigorously computed while, following (43), the second 
contribution can be estimated using

$$
\begin{aligned}
\sum_{\substack{k_{1}+k_{2}=k \\
\left|k_{2}\right|>k+m_{\gamma}}} w_{k_{1}}^{-s^{\star}} w_{k_{2}}^{-s} & =\sum_{k_{2}=k+m_{\gamma}+1}^{\infty}\left[\frac{1}{w_{k_{2}}^{s^{\star}}-k}+\frac{1}{w_{k_{2}+k}^{s^{\star}}}\right] \frac{1}{k_{2}^{s}} \\
& \leq \frac{1}{\left(m_{\gamma}+1\right)^{s^{\star}-s}} \sum_{k_{2}>k+m_{\gamma}}\left[\frac{1}{w_{k_{2}-k}^{s}}+\frac{1}{w_{k_{2}+k}^{s}}\right] \frac{1}{k_{2}^{s}} \\
& \leq \frac{1}{\left(m_{\gamma}+1\right)^{s^{\star}-s}}\left[\sum_{k_{2}=m_{\gamma}+1}^{k+m_{\gamma}} \frac{1}{\left(k_{2}+k\right)^{s}} \frac{1}{k_{2}^{s}}+2 \zeta\left(k+m_{\gamma}+1,2 s\right)\right] .
\end{aligned}
$$

Summarizing, for those $k \geq m, k<M$ such that $k+m_{\gamma}>M$, we define

$$
\begin{array}{r}
H_{k}=\sum_{\substack{k_{1}+k_{2}=k \\
M \leq\left|k_{2}\right| \leq k+m_{\gamma}}}\left(\left|\mathcal{A}_{k_{1}, 1}\right|+\left|\mathcal{A}_{k_{1}, 2}\right|\right) w_{l}^{-s} \mathbb{1}_{n}+\frac{2 n r_{\gamma}}{\left(m_{\gamma}+1\right)^{s^{\star}-s}}\left[\sum_{k_{2}=m_{\gamma}+1}^{k+m_{\gamma}} \frac{1}{\left(k_{2}+k\right)^{s}} \frac{1}{k_{2}^{s}}\right. \\
\left.+2 \zeta\left(k+m_{\gamma}+1,2 s\right)\right] \mathbb{1}_{n}
\end{array}
$$

where, clearly, the dimension of the dynamical system is $n=3$. Again, the knowledge of the particular behavior of the coefficients $\mathcal{A}_{k}$ allows to provide a better estimate for $Z_{M}$. Indeed note that $\left|\mathcal{A}_{k, 1}\right| \leq_{c w}\left|\overline{\mathcal{A}}_{k, 1}\right|+w_{k}^{-s^{\star}} \mathbb{1}_{n}$, where $\overline{\mathcal{A}}_{k}$ denotes the matrix $\mathcal{A}_{k}$ with the entries $\bar{\xi}$ in place of $\xi$ and the same holds for $\left|\mathcal{A}_{k, 2}\right|$. It follows that $\left.\left|\mathcal{A}_{k, 1}\right|_{\infty}+\mid \mathcal{A}_{k, 2}\right)\left.\right|_{\infty}<$ $\sqrt{2}\left|\bar{\xi}_{k}\right|_{\infty}+2 r_{\gamma} w_{k}^{-s^{\star}}$ for $1 \leq|k| \leq m_{\gamma}$ and (56) for $|k|>m_{\gamma}$.

Therefore, the computation of the bound for $\left|c_{k, 1}\right|_{\infty}$ when $k \geq M$, necessary for the definition of $Z_{M}$, has been slightly modified as follows.

$$
\begin{aligned}
\left|c_{k, 1}\right| & =\left|\sum_{\substack{k_{1}+k_{2}=k \\
\left|k_{2}\right| \neq k}}\left(\mathcal{A}_{k_{1}, 1}+\mathcal{A}_{k_{1}, 2}\right) w_{k_{2}}^{-s} \mathbb{1}_{n}\right| \leq_{c w} \sum_{\substack{k_{1}+k_{2}=k \\
k_{1} \neq 0,2 k}}\left(\left|\mathcal{A}_{k_{1}, 1}\right|+\left|\mathcal{A}_{k_{1}, 2}\right|\right) w_{k_{2}}^{-s} \mathbb{1}_{n} \\
& \leq \sum_{\substack{k_{1}+k_{2}=k \\
k_{1} \neq 0,\left|k_{1}\right| \leq m_{\gamma}}}\left(\left|\overline{\mathcal{A}}_{k_{1}, 1}\right|+\left|\overline{\mathcal{A}}_{k_{1}, 2}\right|\right) w_{k_{2}}^{-s} \mathbb{1}_{n}+2 r_{\gamma} \sum_{\substack{k_{1}+k_{2}=k \\
\left|k_{2}\right| \neq k}} w_{k_{1}}^{-s^{\star}} w_{k_{2}}^{-s} \mathbb{1}_{n} \mathbb{1}_{n} .
\end{aligned}
$$

Then, using the fact that $s^{\star} \geq s$, for any $k \geq M$

$$
\begin{aligned}
\left|c_{k, 1}\right|_{\infty} & \leq_{c w} n \sqrt{2} \sum_{j=1}^{m_{\gamma}}\left|\bar{\xi}_{j}\right|_{\infty}\left(w_{k-j}^{-s}+w_{k+j}^{-s}\right)+2 n r_{\gamma} \sum_{\substack{l+j=k \\
|l| \neq k}} w_{j}^{-s} w_{l}^{-s} \\
& \leq_{c w} \frac{n}{k^{s}}\left[\sqrt{2} \sum_{j=1}^{m_{\gamma}}\left|\bar{\xi}_{j}\right|_{\infty} k^{s}\left(w_{k-j}^{-s}+w_{k+j}^{-s}\right)+2 r_{\gamma}\left[1+2 \sum_{l=1}^{M} \frac{1}{l^{s}}+\frac{2}{M^{s-1}(s-1)}+\eta_{M}\right]\right] \\
& \leq_{c w} \frac{n}{k^{s}}\left[\sqrt{2} \sum_{j=1}^{m_{\gamma}}\left|\bar{\xi}_{j}\right|_{\infty}\left(\frac{1}{\left(1-\frac{j}{M}\right)^{s}}+1\right)+2 r_{\gamma}\left[1+2 \sum_{l=1}^{M} \frac{1}{l^{s}}+\frac{2}{M^{s-1}(s-1)}+\eta_{M}\right]\right] .
\end{aligned}
$$




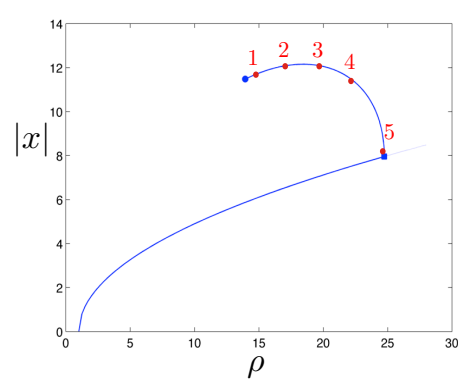

(a)

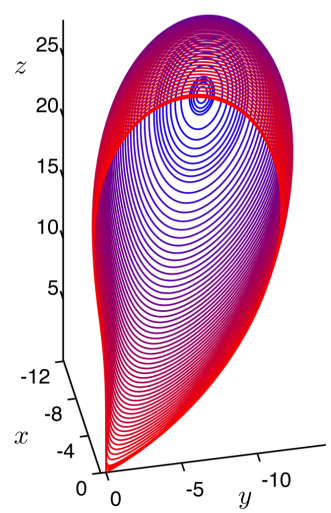

(b)

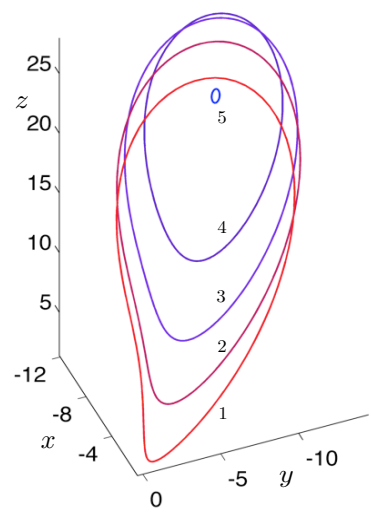

(c)

Figure 2: (a) A simple bifurcation diagram for the Lorenz equations (52). The labelled points correspond to the values $\rho=\rho_{i}(i=1,2, \ldots, 5)$. (b) Some of the rigorously computed periodic orbits on the branch joining the Hopf bifurcation and the homoclinic point. (c) The periodic solutions corresponding to $\rho=\rho_{i}(i=1, \ldots, 5)$.

\subsubsection{Computational results}

For the choice $\sigma=10, \beta=8 / 3$ it is known that there exists a branch of periodic solutions parametrized by $\rho$ joining a Hopf bifurcation at $\rho=\frac{470}{19} \approx 24.736$ and a homoclinic point at $\rho \approx 13.9265$, see Figure 2(a). In what follows, we restrict our attention to the periodic solutions lying on this branch. Table 1 presents data about the computer-assisted proofs of existence of several periodic orbits in the form (53) with bounds of the form (54). As mentioned previously, the existence of such solutions are done via the radii polynomials. For each rigorous computation, we fixed $s^{\star}=2$. Each line of Table 1 corresponds to a different solution and reports the value of $\rho$, the period $\bar{\tau}_{\gamma}$ of the numerical solution, the dimension $m_{\gamma}$ of the finite dimensional projection and the radius $r_{\gamma}$ of the ball $B_{\bar{\gamma}}\left(r_{\gamma}\right)=$ $\bar{\gamma}+\prod_{k \geq 0}\left[-\frac{r_{\gamma}}{w_{k}^{2}}, \frac{r_{\gamma}}{w_{k}^{2}}\right]^{6}$ around the numerical solution $\bar{\gamma}$ where the exact solution has been proved to exist. Some of the periodic orbits are depicted in Figure 2(b). Note that with the radii polynomials, it is possible to rigorously enclose the periodic solutions for values of $\rho$ close to the Hopf bifurcation and to the homoclinic point. As $\rho$ approaches the homoclinic point, the periodic solutions are flatter (e.g. see Figure 3), which means that a larger number of Fourier coefficients contributes to their Fourier expansions. Therefore, it is necessary to chose a larger finite dimensional projection $m_{\gamma}$ as $\rho$ decreases in order to obtain rigorous computations of existence of periodic orbits using the radii polynomials.

The rigorous computation of the enclosure of the invariant bundles was done for a set of periodic orbits lying on the same bifurcation branch (see Figure 2(a)), and the corresponding values of $\rho$ are

$$
\rho_{1}=14.85, \quad \rho_{2}=17.32, \quad \rho_{3}=19.79, \quad \rho_{4}=22.26, \quad \rho_{5}=24.73 .
$$

Figure 2(c) contains the plot of five periodic orbits $\bar{\gamma}_{i}(i=1, \ldots, 5)$ at $\rho=\rho_{i}(i=1, \ldots, 5)$, 

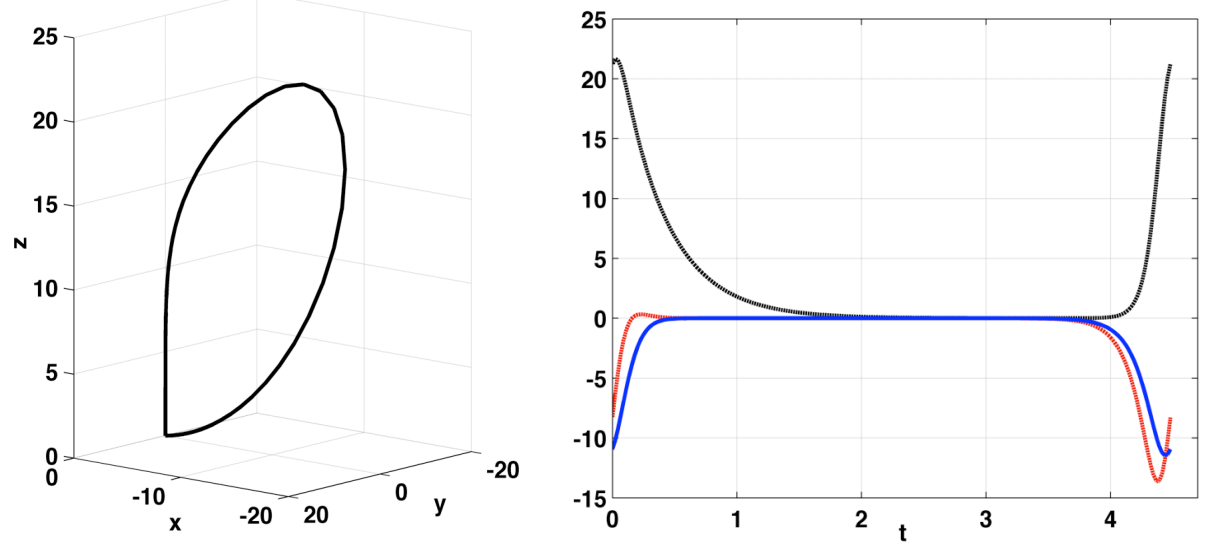

Figure 3: Periodic orbit at $\rho=13.927$. On the left, the orbit is drawn in the state space, while on right the $x$ (blue), $y$ (red), $z$ (black) coordinates are plotted separately as function of time. A genuine solution has been proved to exist with Fourier coefficients in a ball of radius $r=1.893591107536733 \cdot 10^{-08}$ around the numerical solution.

\begin{tabular}{cccc}
$\rho$ & $\bar{\tau}_{\gamma}$ & $m_{\gamma}$ & $r_{\gamma}$ \\
\hline 24.736 & 0.652859396941149 & 30 & $9.469873202388920 \cdot 10^{-11}$ \\
24.436 & 0.663384674511011 & 30 & $5.478345151088000 \cdot 10^{-12}$ \\
23.200 & 0.710825719902523 & 40 & $3.071478394415574 \cdot 10^{-12}$ \\
20.800 & 0.827536078261055 & 40 & $1.857127168671614 \cdot 10^{-12}$ \\
16.000 & 1.302497474229172 & 40 & $2.574513942375312 \cdot 10^{-12}$ \\
15.400 & 1.435095007378964 & 40 & $1.178559327141313 \cdot 10^{-11}$ \\
14.900 & 1.594009133698383 & 48 & $4.951779335080891 \cdot 10^{-12}$ \\
14.300 & 1.955959084775736 & 48 & $5.924372707046972 \cdot 10^{-10}$ \\
14.100 & 2.243447758187898 & 56 & $3.426711644299483 \cdot 10^{-10}$ \\
14.000 & 2.565212920927481 & 56 & $2.018825516161773 \cdot 10^{-08}$ \\
13.980 & 2.684270226581966 & 60 & $1.090913664984519 \cdot 10^{-08}$ \\
13.960 & 2.859882454655782 & 60 & $6.320026130098352 \cdot 10^{-08}$ \\
13.940 & 3.201446568839638 & 60 & $1.009627398529485 \cdot 10^{-06}$ \\
13.932 & 3.540414720761810 & 60 & $8.496363668377804 \cdot 10^{-06}$ \\
13.928 & 4.038292895738813 & 80 & $3.658487332536346 \cdot 10^{-07}$ \\
13.927 & 4.481359736174591 & 100 & $1.893591107536733 \cdot 10^{-08}$
\end{tabular}

Table 1: Data associated to the computer-assisted proofs of existence (done with the radii polynomials) of several periodic orbits of the Lorenz equations (52) at different parameter values. 

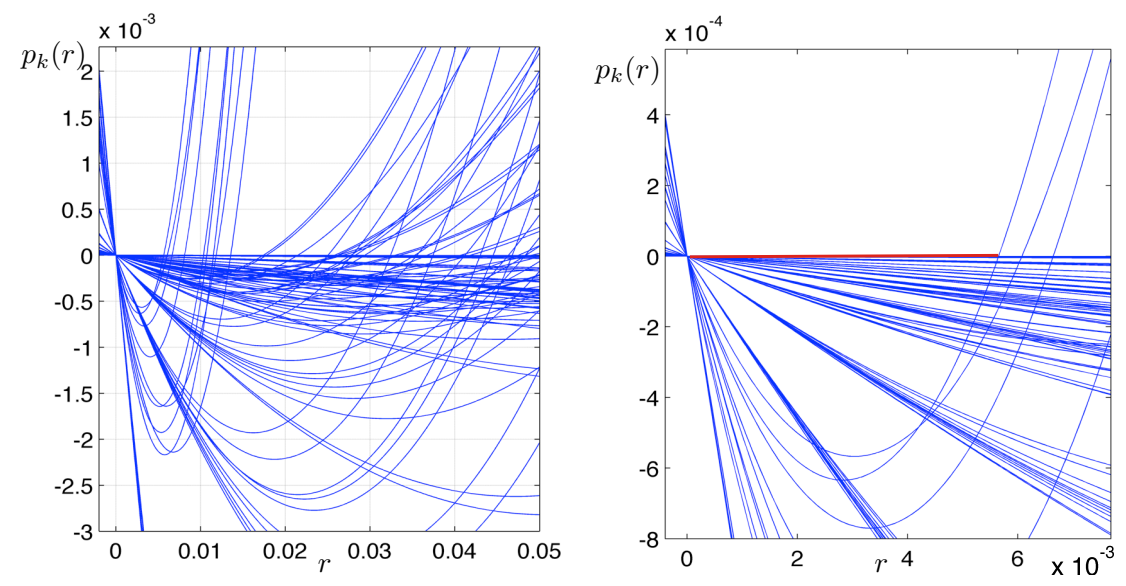

Figure 4: Plot of the radii polynomials $p_{k}(r)$ constructed for the rigorous computation of the Floquet normal form of the fundamental matrix solution associated to the periodic solution \#4 given by $\gamma_{4}$. On the right: a magnification of the radii polynomials close to $r=0$. The red line denotes the interval INT given in (62), where all the $p_{k}(r)$ are negative.

and the Appendix contains the first 15 Fourier coefficients of $\bar{\gamma}_{1}$ and $\bar{\gamma}_{4}$. Table 2 contains the data of the rigorous computation of the Floquet normal forms of the fundamental matrix solutions associated to $\bar{\gamma}_{1}$ and $\bar{\gamma}_{4}$. More precisely, it contains the dimension $m$ of the finite dimensional projection, the computational parameter $M$ and the enclosing radius $r$.

\begin{tabular}{c|c|c|c|c}
$\#$ sol & $\rho$ & $m$ & $M$ & $r$ \\
\hline 1 & 14.85 & 180 & 900 & $1.734418508431413 \cdot 10^{-06}$ \\
2 & 17.32 & 90 & 180 & $3.914300837825743 \cdot 10^{-09}$ \\
3 & 19.79 & 70 & 120 & $3.157918269225057 \cdot 10^{-10}$ \\
4 & 22.26 & 60 & 100 & $1.664781163947245 \cdot 10^{-10}$ \\
5 & 24.73 & 40 & 80 & $1.404020127017372 \cdot 10^{-09}$
\end{tabular}

Table 2: Data associated to the rigorous computation of the Floquet normal form of the fundamental matrix solution for each of the periodic orbit $\gamma_{i}(i=1, \ldots, 5) . m$ is the dimension of the finite dimensional projection, $M$ is the computational parameter and $r$ is the radius of the ball centered at the approximate solution in $\Omega^{s}$ within which a genuine solution of (6) exists.

Some of the radii polynomials $p_{k}(r)$ built during the rigorous computation of the Floquet normal form of the fundamental matrix solution associated to the periodic orbit $\bar{\gamma}_{4}$ are plotted in Figure 4. The bold line on the $r$-axis corresponds to the interval

$$
I N T=\left[1.794002077820062 \cdot 10^{-10} \quad 0.005654240115476\right]
$$

where all the radii polynomials are negative.

From the rigorous computations of the Floquet normal form, we noticed that the odd Fourier coefficients of $Q(t)$ are almost vanishing, suggesting that $Q(t)$ is a $\tau_{\gamma}$-periodic function, rather than $2 \tau_{\gamma}$-periodic. This is not in contradiction with Floquet Theorem. In the Appendix, we present the numerical approximation $\bar{R}$ and the first even Fourier coefficients $\overline{\mathcal{Q}}_{k}$ for the solutions \#1 and \#4. Since the Fourier coefficients $\overline{\mathcal{Q}}_{k}$ corresponding to a pe- 
riodic orbit close to the homoclinic orbit decrease slower, larger values of $m$ and $M$ were necessary to obtain successful computations.

We now have all the ingredients necessary to construct the tangent bundles: first we compute the intervals containing the spectrum and the eigenvectors the interval value matrix $R$, then, in light of Theorem 3.3, the multiplication of the stable and unstable directions (given by the eigenvectors of $R$ ) with the function $Q(\theta)$ yields rigorously the tube enclosing the complete stable and unstable bundles. As already mentioned above, the codes necessary to compute the Floquet normal forms for the Lorenz equations can be found in [29].

\begin{tabular}{r|rl} 
Sol \# & \multicolumn{1}{|c}{ Center } & \multicolumn{1}{c}{ Radius } \\
\hline 1 & -15.109380514113965 & $6.535796640191043 \cdot 10^{-6}$ \\
& 1.4427138474477833 & $2.445722953735543 \cdot 10^{-4}$ \\
\hline 2 & -14.418434214853773 & $1.581998979110051 \cdot 10^{-8}$ \\
& 0.751767548187609 & $2.292988975871850 \cdot 10^{-7}$ \\
\hline 3 & -14.075379469563303 & $1.120430189449109 \cdot 10^{-9}$ \\
& 0.408712802896790 & $1.443149018164319 \cdot 10^{-8}$ \\
\hline 4 & -13.840311255652775 & $4.525292794068323 \cdot 10^{-10}$ \\
& 0.173644588986231 & $6.273796862526999 \cdot 10^{-9}$ \\
\hline 5 & -13.667080400789002 & $4.104230777149636 \cdot 10^{-9}$ \\
& 0.000413734122254 & $9.998501090433916 \cdot 10^{-6}$
\end{tabular}

Table 3: Lyapunov exponents for each of the periodic orbit $\gamma_{i}(i=1, \ldots, 5)$. For each solution, we report the center and the radius of the interval vectors enclosing the exponents. Note that we could prove the existence of the eigenvectors $v_{j}$ associated to $\mu_{j}$ within accuracy given by $r$.

Table 3 lists the Lyapunov exponents of the periodic orbits, as defined in Definition 5, and it also contains the radii of the intervals enclosing the stable and unstable eigen-couple of $R$ while in Figure 5 the tangent bundles are depicted. In Appendix the complete list of the eigen-decomposition of the interval matrices $R$ is also provided.

Before closing this section, let us make few remarks. The radii $r$ of the ball $B_{\bar{x}}(r, s)$ showed in Table 2 come as result of different ingredients which are in some sense in competition. First of all the accuracy of the enclosure of the Floquet normal form is limited by the enclosure of the periodic orbit $\gamma(t)$ : if $s=s^{\star}$ one can not expect the radius $r$ to be smaller then the radius $r_{\gamma}$. Moreover the radius $r$ of the ball $B_{\bar{x}}(r, s)$ in $\Omega^{s}$, where the existence of the genuine solution is proved to exist, depends on how close the numerical approximation is to the real solution. As already said, the definition of the numerical solution $\left(\bar{R}, \overline{\mathcal{Q}}_{k}\right)$ comes from the combination of two numerical integrations, from where we extract $\mathscr{R}$ and $\mathscr{Q}_{k}$, and the implementation of a Newton scheme to find the zeros of the finite dimensional problem $f^{(m)}=0$. For the first we adopted a variable time step Runge Kutta method of fourth order, as implemented in the built in Matlab function ode45, while the Newton scheme is run until $\left|f^{(m)}\right|_{\infty}<2 \cdot 10^{-14}$. Finally, the performance of $r$ is given by the choice of the finite dimensional parameter $m$ and the computational parameter $M$. While the first addresses a theoretical issue and fixes the dimension of the Garlerkin projection, the second serves to better estimate the various bounds necessary in the proof. If on one side a choice of large values for $m$ and $M$ decreases the analytical tail errors, on the other side it increases the number of computations and therefore the error propagation and the computational time. Thus the best result is often given as a tradeoff between this two competitors. 

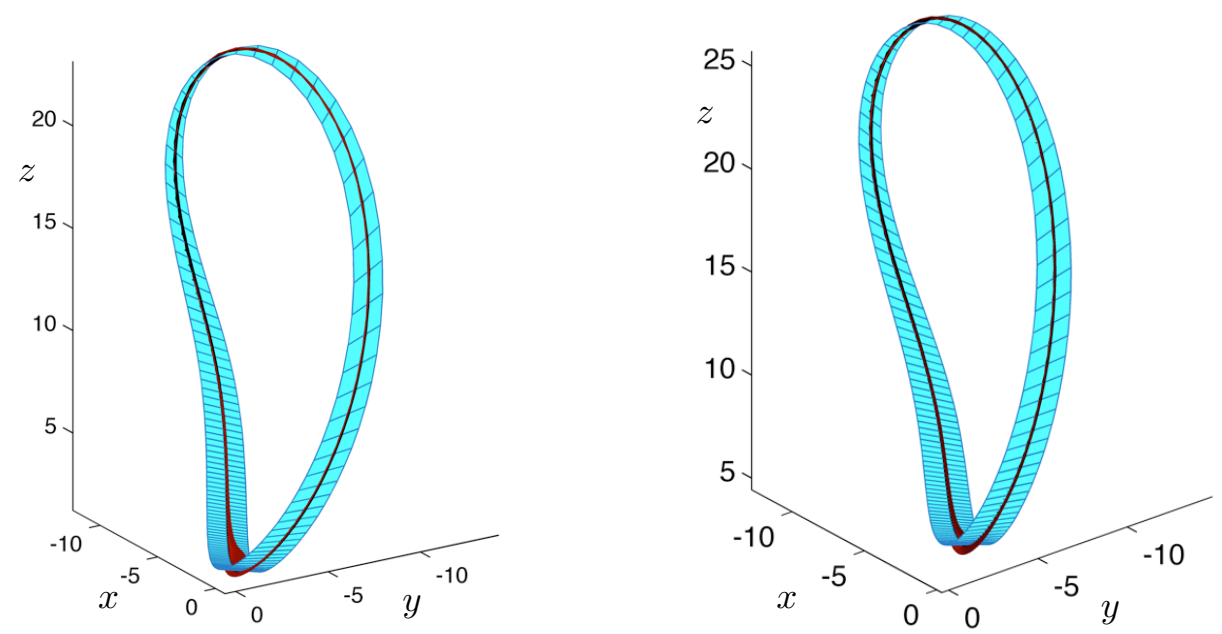

(a)

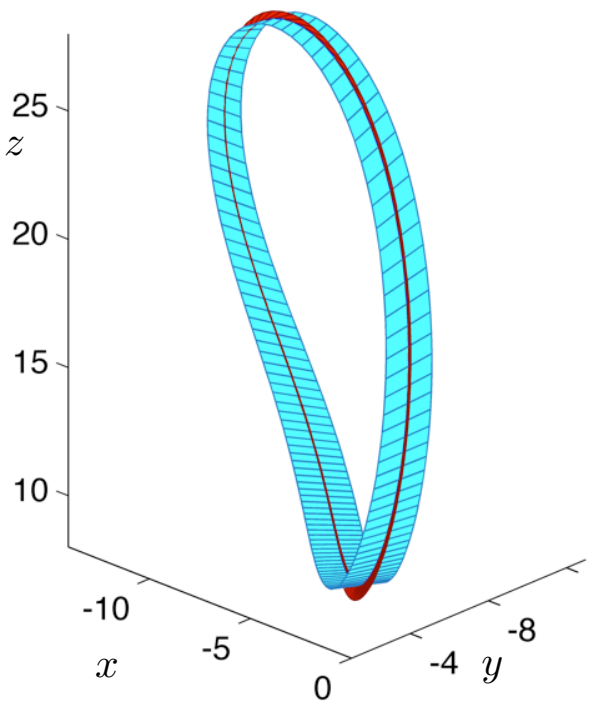

(b)

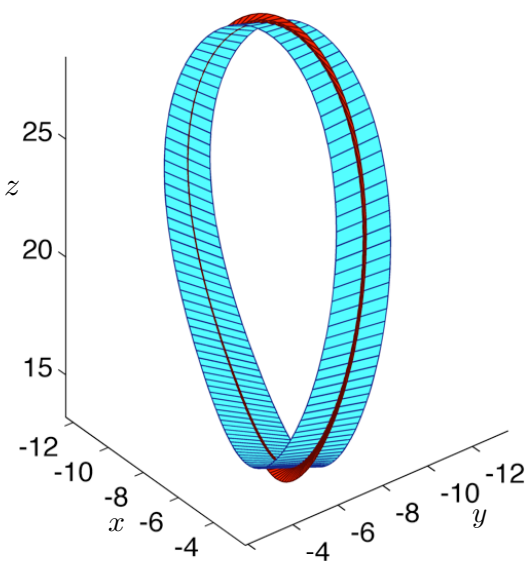

(d)

(c)

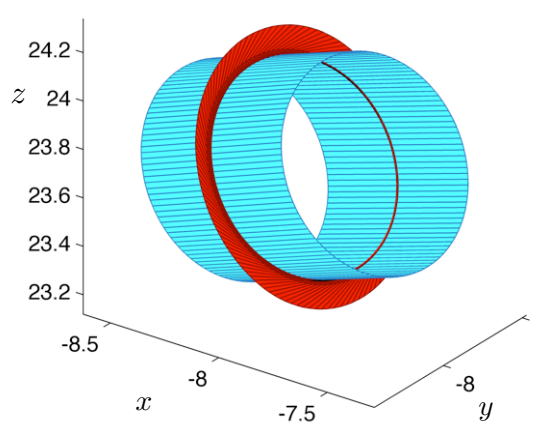

(e)

Figure 5: Plot of the tangent stable (turquoise) and unstable (red) bundles of each of the periodic orbits $\gamma_{i}$. Figures (a)-(b)-(c)-(d)-(e) concern respectively $\gamma_{1}, \gamma_{2}, \gamma_{3}, \gamma_{4}, \gamma_{5}$. 


\subsection{The $\zeta^{3}$-model: non orientable tangent bundles}

It is known that if a Floquet multiplier of a periodic orbit is negative, then the corresponding tangent bundle is not orientable. Moreover, in the case of a saddle periodic orbit of a threedimensional system, the two non-trivial Floquet multipliers are real and their product is positive. Therefore both the tangent bundles are either orientable or not orientable and, in the latter case, they are topologically equivalent to a Möbius strip, see [26].

An example of a dynamical system with periodic orbits that exhibit this behavior is the so called $\zeta^{3}$-model considered in [27]

$$
\left\{\begin{array}{l}
\dot{x}=y \\
\dot{y}=z \\
\dot{z}=\alpha x-x^{2}-\beta y-z
\end{array}\right.
$$

For $\beta=2$, as $\alpha$ varies, the periodic orbits of system (63) produce an interesting bifurcation diagram. We refer to [26] and [28] for a detailed analysis of the bifurcation diagram and on the genesis of periodic orbits, called twisted periodic orbits, with non orientable invariant manifolds. We focus on a particular twisted periodic orbit corresponding to $\alpha=3.372$ lying on the branch emanating from a period-doubling bifurcation that occurs at $\alpha \approx 3.125$.

Following the same procedure as before, we rigorously compute the enclosure of the periodic orbit $\gamma(t)$ and subsequently the enclosure of the matrix $R$ and of the matrix function $Q(t)$, hence producing an explicit Floquet normal form as in (2). Then, we extract the necessary stability parameters and we recover the stable and unstable tangent bundles using (51). Figure 6 shows the resulting bundles.

For the rigorous computation of the periodic orbit we chose $m_{\gamma}=30$ proving the genuine periodic orbit to live in a ball of radius $r_{\gamma}=1.657473362439634 \cdot 10^{-12}$ around the numerical approximation, $\left(s^{\star}=2\right)$, while for the enclosure of the Floquet normal form of the principal fundamental matrix solution of the linearized problem we set $m=50, M=100$ yielding the enclosure radius $r=8.327055238174269 \cdot 10^{-10}$. Having computed the intervals enclosing the period $\tau$ of the orbit and the eigenvalues of $R$, we realize that the absolute values of the two nontrivial Floquet multipliers satisfy

$$
\begin{aligned}
& \left|\sigma_{1}\right| \in\left[\begin{array}{ll}
0.007038336031738 & 0.007038336266547
\end{array}\right] \\
& \left|\sigma_{2}\right| \in\left[\begin{array}{ll}
1.527362891655825 & 1.527363232279426
\end{array}\right]
\end{aligned} .
$$

To conclude we emphasize the role played by the continuous function $Q(\theta)$ in the construction of the tangent bundles. As proved in Theorem 3.3, as $\theta$ changes, the eigenvector $w_{j}^{\theta}$ of $\Phi_{\theta}(\tau)$ associated to the Floquet multiplier $\sigma_{j}$ is given by $w_{j}^{\theta}=Q(\theta) v_{j}$, where $v_{j}$ is the eigenvector of $R$ relative to the eigenvalue $\mu_{j}$. The function $Q(\theta)$ is continuous and $2 \tau$-periodic, but the tangent bundles are smooth manifolds, therefore the eigenspaces $E_{s}^{\theta}$ and $E_{u}^{\theta}$, as function of $\theta \in[0,2 \tau]$, must be a double covering. That implies that $w_{j}^{\tau}=Q(\tau) v_{j}$ has to be an eigenvector of $\Phi(\tau)$ associated to the Floquet multiplier $\sigma_{j}$, i.e. $\operatorname{span}\left\{v_{j}\right\}=\operatorname{span}\left\{w_{j}^{\tau}\right\}$. In the case of the Lorenz equations $(52), Q(\tau)$ turns to be the identity matrix, therefore the last relation is simply verified. But in case of the $\zeta^{3}$-model and in general when the bundle is not orientable, $Q(\tau)$ need not be the identity matrix. Indeed, in the considered example, $Q(\tau)$ results to stay in a small interval around

$$
\bar{Q}=\left[\begin{array}{rrr}
-1.675372218349393 & -1.030485782114017 & -0.456425794029489 \\
1.323704549546922 & 1.019713990251906 & 0.894577662024640 \\
0.970176001842336 & 1.480298639600749 & -0.344341771902499
\end{array}\right]
$$

and the relation $\operatorname{span}\left\{v_{j}\right\}=\operatorname{span}\left\{w_{j}^{\tau}\right\}$ still holds. 


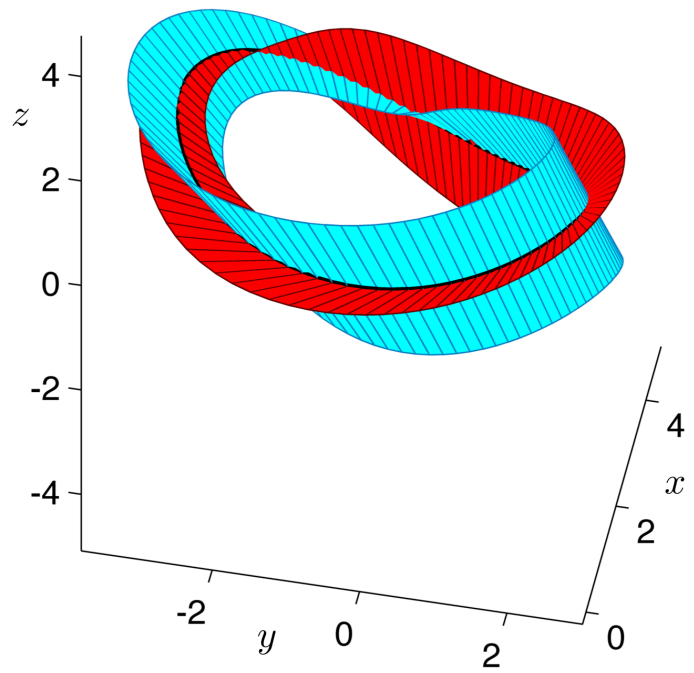

(a)

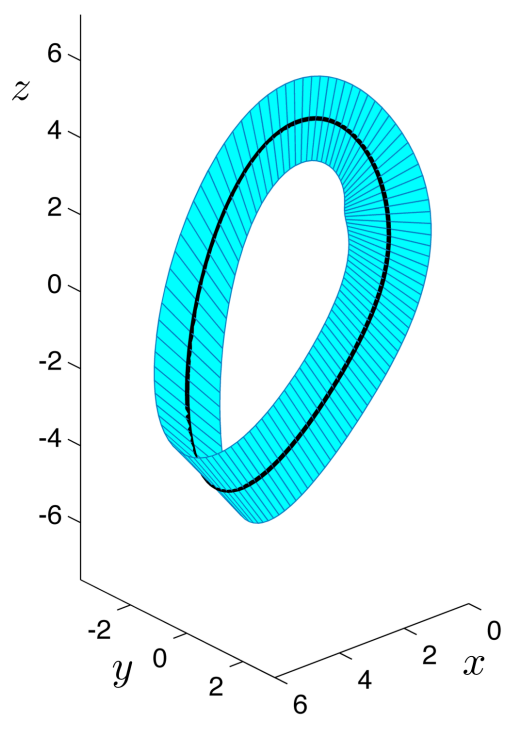

(b)

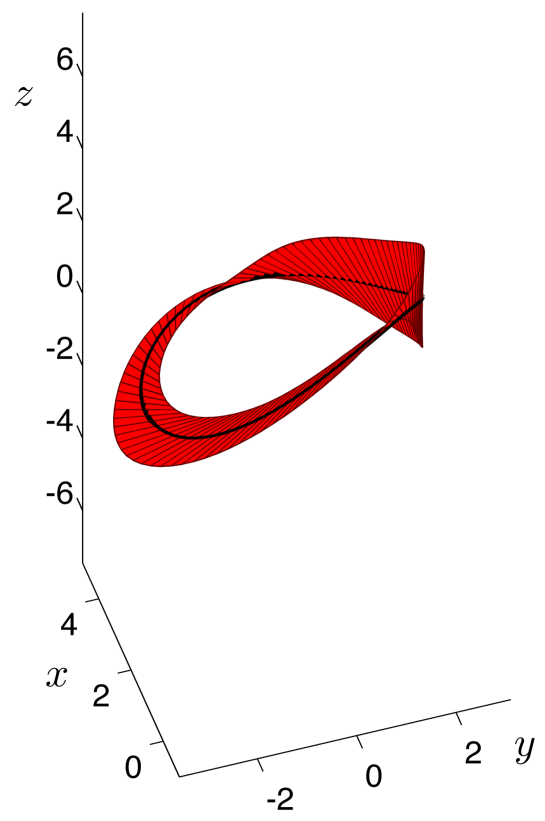

(c)

Figure 6: Rigorously computed stable (turquoise) and unstable (red) tangent bundles of a periodic orbit of the $\zeta^{3}$ model with negative Floquet multipliers 


\subsection{Recovering of the Floquet multipliers}

In this final section, we discuss how to recover a posteriori the Floquet multipliers associated to the periodic orbit $\gamma(t)$, that is the eigenvalues of the monodromy matrix $\Phi_{\theta}(\tau)$. First recall from Lemma 3.2 that the Floquet multipliers, denoted by $\sigma_{j}$, are independent of $\theta$ and from Theorem 3.3 that they solve the equation

$$
\Phi_{\theta}(\tau) w_{j}^{\theta}=\sigma_{j} w_{j}^{\theta}
$$

for any $\theta$. In the proof of Theorem 3.3 we realized that $\sigma_{j}^{2}=e^{\mu_{j} 2 \tau}$ where $\mu_{j}$ are the eigenvalues of the matrix $R$. Thus the Floquet multipliers are known up to a sign, i.e. $\sigma_{j}= \pm e^{\mu_{j} \tau}$. Therefore, by choosing $\theta=0$ in (64), it is enough to check whether $\Phi(\tau) w_{j}^{0}$ is equal to $+e^{\mu_{j} \tau} w_{j}^{0}$ or to $-e^{\mu_{j} \tau} w_{j}^{0}$. Recall that the Floquet normal form $\Phi(t)=Q(t) e^{R t}$ satisfies $Q(0)=I_{n}$ and recall from (51) that $w_{j}^{\theta}:=Q(\theta) v_{j}$. Hence, since $\left(\mu_{j}, v_{j}\right) \in \Sigma(R)$, one has that $\Phi(\tau) w_{j}^{0}=Q(\tau) e^{R \tau} v_{j}=Q(\tau) e^{\mu_{j} \tau} v_{j}$. Thus it reduces to compute the vectors $Q(\tau) e^{\mu_{j} \tau} v_{j}$ and $e^{\mu_{j} \tau} v_{j}$ and to compare them.

Having computed the enclosure of $Q(t)$ in terms of the Fourier coefficients and the bounds for $\mu_{j}$ and $v_{j}$, denote by $\mathcal{I}_{\sigma_{j}}$ the interval enclosing $Q(\tau) e^{\mu_{j} \tau} v_{j}$ and by $\mathcal{I}_{j}^{+}, \mathcal{I}_{j}^{-}$the intervals enclosing $e^{\mu_{j} \tau} v_{j}$ and $-e^{\mu_{j} \tau} v_{j}$, respectively. The sign of the Floquet multiplier $\sigma_{j}$ is determined if $\mathcal{I}_{\sigma_{j}}$ intersects only one of the intervals $\mathcal{I}_{j}^{+}$and $\mathcal{I}_{j}^{-}$, while no conclusion can be achieved if $\mathcal{I}_{\sigma_{j}}$ is so large to intersect both of them. Note that $\mathcal{I}_{\sigma_{j}}$ has to intersect at least one $\mathcal{I}_{j}^{ \pm}$. The choice $\theta=0$ in (64) increases the chances of successfully recovering the Floquet multipliers since in this case, the computation of the matrix exponentiation $e^{R \tau}$, a computation that can dramatically increase the error propagation, can be avoided.

Finally, Table 4 and Table 5 contain the intervals $\mathcal{I}_{\sigma_{j}}$ and $\mathcal{I}_{j}^{+}, j=1,2$ associated to the solution \#3 of the Lorenz equations and of the solution of the $\zeta^{3}$-model. That shows that the Floquet multipliers $\sigma_{1}, \sigma_{2}$ are both positive for the periodic solution of the Lorenz equations and are both negative for the periodic solution the $\zeta^{3}$-model.

Solution Lorenz \#3

$\left|\sigma_{2}\right| \in[0.3599654189589730 .359965419696942] \cdot 10^{-5}$

$\left|\sigma_{1}\right| \in\left[\begin{array}{lll}1.439037708132836 & 1.439037745123575\end{array}\right]$

\begin{tabular}{|c|c|}
\hline $\mathcal{I}_{\sigma_{1}}$ & $\mathcal{I}_{1}^{+}$ \\
\hline $10^{-5}$ & $10^{-5}$ \\
\hline 0.2756432688658180 .275643271985182 & 0.2756432697396350 .275643271111366 \\
\hline 0.0650769194810430 .065076922168721 & 0.0650769203548610 .065076921294908 \\
\hline 0.2221730953296390 .222173098339382 & 0.2221730962034590 .222173097465571 \\
\hline
\end{tabular}

$\mathcal{I}_{\sigma_{2}}$ $\mathcal{I}_{2}^{+}$

$\left[\begin{array}{lll}-0.263299353291831 & -0.263299298199846 \\ -1.237252678484705 & -1.237252598357060 \\ -0.686082354892901 & -0.686082288933197\end{array}\right]\left[\begin{array}{lll}-0.263299349897387 & -0.263299301594310 \\ -1.237252675090274 & -1.237252601751541 \\ -0.686082351498413 & -0.686082292327618\end{array}\right]$

Table 4: Interval vectors $\mathcal{I}_{\sigma_{j}}$ and $\mathcal{I}_{j}^{+}, j=1,2$ associated to sol\#3 for the Lorenz equations (52). It holds $\mathcal{I}_{j}^{+} \subset \mathcal{I}_{\sigma_{j}}, j=1,2$ proving that the Floquet multipliers are positive 
Solution $\zeta^{3}$-model

$\left|\sigma_{1}\right| \in[0.007038336031738 \quad 0.007038336266547]$

$\left|\sigma_{2}\right| \in[1.527362891655825 \quad 1.527363232279426]$

$\frac{\mathcal{I}_{\sigma_{1}}}{\left[\begin{array}{rr}0.001505025554611 & 0.001505026041576 \\ 0.001935764317690 & 0.001935764886660 \\ -0.006597415507817 & -0.006597415070914\end{array}\right]\left[\begin{array}{rr}-0.001505025849095 & -0.001505025747089 \\ -0.001935764660362 & -0.001935764543986 \\ 0.006597415153419 & 0.006597415425314\end{array}\right]}$

Table 5: Interval vectors $\mathcal{I}_{\sigma_{j}}$ and $\mathcal{I}_{j}^{+}, j=1,2$ associated to solution of the $\zeta^{3}$-model. It holds $-\mathcal{I}_{j}^{+}=\mathcal{I}_{j}^{-} \subset \mathcal{I}_{\sigma_{j}}, j=1,2$ proving that the Floquet multipliers are negative.

\section{Acknowledgments}

We would like to thank Marcio Gameiro and Jason D. Mireles James for helpful discussions.

\section{Appendix}

\subsection{Computation of the constant $C_{\Lambda}$ of Lemma 2.2}

The construction of $C_{\Lambda}$ is done by bounding uniformly the right hand side of (18) for $k \geq M$ up to a factor $1 / k$. For this it is convenient to write explicitly the entries of the matrix $\hat{\Lambda}$ appearing in (17)

$$
\hat{\Lambda}_{k}:=\left[\begin{array}{cc}
k \frac{2 \pi}{2 \tau} I_{n^{2}}+\lambda_{2,1} & \lambda_{2,2} \\
\lambda_{1,1} & -k \frac{2 \pi}{2 \tau} I_{n^{2}}+\lambda_{1,2}
\end{array}\right] .
$$

A straightforward calculation implies that

$$
\lambda_{1,1}=R^{*}-\left[\mathcal{A}_{0}\right]_{n}-\left[\mathcal{A}_{2 k, 1}\right]_{n}, \quad \lambda_{2,2}=R^{*}-\left[\mathcal{A}_{0}\right]_{n}+\left[\mathcal{A}_{2 k, 1}\right]_{n}, \quad \lambda_{1,2}=\lambda_{2,1}=-\left[\mathcal{A}_{2 k, 2}\right]_{n},
$$

where

$$
R^{*}:=\left[\begin{array}{ccc}
\bar{R}(1,1) I_{n} & \ldots & \bar{R}(n, 1) I_{n} \\
\vdots & \ddots & \vdots \\
\bar{R}(1, n) I_{n} & \ldots & \bar{R}(n, n) I_{n}
\end{array}\right] \in \operatorname{Mat}\left(n^{2}, \mathbb{R}\right)
$$

and the notation $[\mathcal{B}]_{n}$ stands for the diagonal concatenation of $n$ copies of $\mathcal{B} \in \operatorname{Mat}(n, \mathbb{R})$, i.e.

$$
[\mathcal{B}]_{n}:=\left[\begin{array}{ccc}
\mathcal{B} & & \\
& \ddots & \\
& & \mathcal{B}
\end{array}\right] \in \operatorname{Mat}\left(n^{2}, \mathbb{R}\right) .
$$


We note that, with the exception of the factors $k \frac{2 \pi}{2 \tau}$ on the diagonal, the matrix $\hat{\Lambda}_{k}$ depends on $k$ only through the entries of the matrices $\mathcal{A}_{2 k, 1}$ and $\mathcal{A}_{2 k, 2}$. The hypothesis $\|\mathcal{A}\|_{s^{*}}<\infty$ implies that

$$
\left|\mathcal{A}_{2 k, 1}\right|_{\infty},\left|\mathcal{A}_{2 k, 2}\right|_{\infty} \leq\left|\mathcal{A}_{2 k}\right|_{\infty} \leq \frac{\|\mathcal{A}\|_{s^{*}}}{(2 k)^{s^{*}}} \leq \frac{\|\mathcal{A}\|_{s^{*}}}{(2 M)^{s^{*}}},
$$

so we have the component-wise estimates

$$
\left|\mathcal{A}_{2 k, 1}\right| \leq_{c w} \frac{\|\mathcal{A}\|_{s^{*}}}{(2 M)^{s^{*}}} \mathbb{1}_{n}, \quad\left|\mathcal{A}_{2 k, 2}\right| \leq_{c w} \frac{\|\mathcal{A}\|_{s^{*}}}{(2 M)^{s^{*}}} \mathbb{1}_{n} .
$$

Therefore the diagonal elements of $\hat{\Lambda}_{k}$ satisfy $\left|\hat{\Lambda}_{k}(i, i)\right| \geq k \frac{2 \pi}{2 \tau}-\frac{\|\mathcal{A}\|_{s^{*}}}{(2 M)^{s^{*}}}$, while the off diagonal terms satisfy $\left|\hat{\Lambda}_{k}(i, j)\right| \leq \bar{\Lambda}_{M}(i, j)$ for every $k \geq M$, where

$$
\bar{\Lambda}_{M}:=\left[\begin{array}{cc}
{\left[\frac{\|\mathcal{A}\|_{s^{*}}}{(2 M)^{*}} \mathbb{1}_{n}\right]_{n}} & \left|R^{*}-\left[\mathcal{A}_{0}\right]_{n}\right|+\left[\frac{\|\mathcal{A}\|_{s^{*}}}{(2 M)^{s^{*}}} \mathbb{1}_{n}\right]_{n} \\
\left|R^{*}-\left[\mathcal{A}_{0}\right]_{n}\right|+\left[\frac{\|\mathcal{A}\|_{s^{*}}}{(2 M)^{s^{*}}} \mathbb{1}_{n}\right]_{n} & {\left[\frac{\|\mathcal{A}\|_{s^{*}}}{(2 M)^{s^{*}}} \mathbb{1}_{n}\right]_{n}}
\end{array}\right] .
$$

Inserting the previous bounds in formula (18), we obtain

$$
\left\|\hat{\Lambda}_{k}^{-1}\right\|_{\infty} \leq \frac{1}{\min _{i}\left\{k \frac{2 \pi}{2 \tau}-\frac{\|\mathcal{A}\|_{s^{*}}}{(2 M)^{s^{*}}}-\sum_{j \neq i} \bar{\Lambda}_{M}(i, j)\right\}} \leq \frac{1}{k \min _{i}\left\{\frac{2 \pi}{2 \tau}-\left(\frac{\|\mathcal{A}\|_{s^{*}}}{(2 M)^{s^{*}}}\right) \frac{1}{k}-\frac{1}{k} \sum_{j \neq i} \bar{\Lambda}_{M}(i, j)\right\}}
$$

and, finally, $\left\|\Lambda_{k}^{-1}\right\|_{\infty}=\left\|\hat{\Lambda}_{k}^{-1}\right\|_{\infty} \leq \frac{C_{\Lambda}}{k}$, for every $k \geq M$, where

$$
C_{\Lambda}:=\frac{1}{\min _{i}\left\{\frac{2 \pi}{2 \tau}-\left(\frac{\|\mathcal{A}\|_{s^{*}}}{(2 M)^{s^{*}}}\right) \frac{1}{M}-\frac{1}{M} \sum_{j \neq i} \bar{\Lambda}_{M}(i, j)\right\}} .
$$

Remark that the computation of $C_{\Lambda}$ depends on the uniform bound of the matrices $\mathcal{A}_{2 k}$ for $k$ larger than $M$. For sake of generality, we considered the weakest hypothesis $\|\mathcal{A}\|_{s^{*}}<\infty$, yielding the estimates (65). However, a more precise information about the behavior of $\mathcal{A}_{2 k}$ allows constructing a sharper $C_{\Lambda}$. For instance, in Section 4.1, a bound slightly sharper than the one given by (67) is obtained in the context of the Lorenz equations.

\subsection{Data}

The period and the Fourier coefficients of $\bar{\gamma}_{1}$ and $\bar{\gamma}_{4}$ :

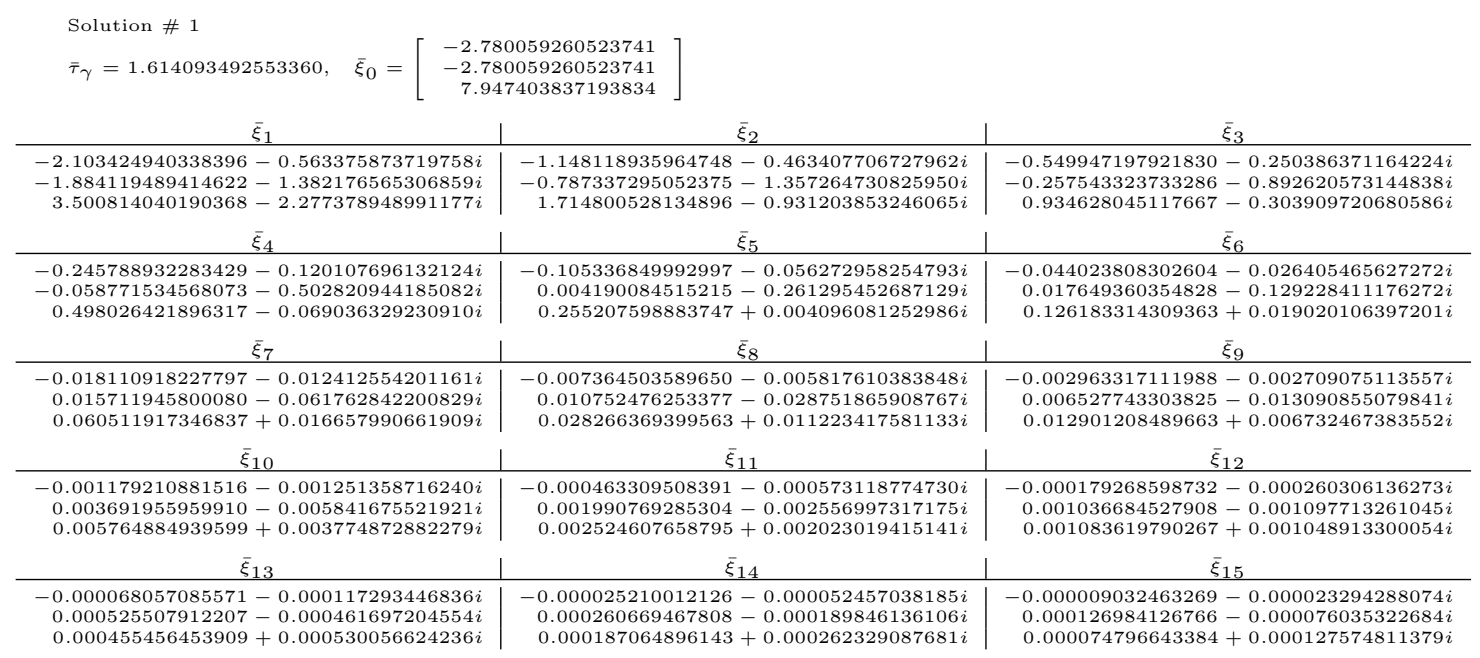


Solution \# 4

$\bar{\tau}_{\gamma}=0.752056588663314, \quad \bar{\xi}_{0}=\left[\begin{array}{r}-6.701197977052439 \\ -6.701197977052439 \\ 19.896645313603468\end{array}\right]$

\begin{tabular}{r|r|r}
\multicolumn{1}{c|}{$\bar{\xi}_{1}$} & \multicolumn{1}{c}{$\bar{\xi}_{2}$} & \multicolumn{1}{c}{$\bar{\xi}_{3}$} \\
\hline$-1.957527201575398-0.358818882759485 i$ & $-0.335512945846433-0.016213444633248 i$ & $-0.047773518005275+0.003264331987478 i$ \\
$-1.657745833247766-1.994268440246074 i$ & $-0.308421346986976-0.576833494637121 i$ & $-0.055955243858351-0.116475274417622 i$ \\
$2.644506821861307-2.227265633430878 i$ & $0.566785859807531-0.332890753309569 i$ & $0.114165757724204-0.053764689555239 i$
\end{tabular}

\begin{tabular}{r|r|r}
$\bar{\xi}_{4}$ & \multicolumn{1}{c}{$\bar{\xi}_{5}$} & \multicolumn{1}{c}{$\bar{\xi}_{6}$} \\
\hline$-0.006558770971049+0.000929180341579 i$ & $-0.000901308891171+0.000177163318124 i$ & $-0.000124174690936+0.000030576566572 i$ \\
$-0.009663969360603-0.020989368884455 i$ & $-0.001641379501671-0.003587906270789 i$ & $-0.000277448981776-0.000591886640616 i$ \\
$0.020940359140456-0.009162879791036 i$ & $0.003602280657498-0.001579182778059 i$ & $0.000594586513762-0.000270108958042 i$
\end{tabular}

\begin{tabular}{r|r|r}
$\bar{\xi}_{7}$ & \multicolumn{1}{c|}{$\bar{\xi}_{8}$} & \multicolumn{1}{c}{$\bar{\xi}_{9}$} \\
\hline$-0.000017112175371+0.000005045265812 i$ & $-0.000002356075416+0.000000809468453 i$ & $-0.000000323960977+0.000000127320510 i$ \\
$-0.000046618250245-0.000095031349885 i$ & $-0.000007766349449-0.000014937919344 i$ & $-0.000001281309839-0.000002308608107 i$ \\
$0.000095408145471-0.000045683567994 i$ & $0.000014990676622-0.000007640211987 i$ & $0.000002316321304-0.000001263950355 i$
\end{tabular}

\begin{tabular}{c|r|r}
$\bar{\xi}_{10}$ & $\bar{\xi}_{11}$ & $\bar{\xi}_{12}$ \\
\hline$-0.000000044474813+0.000000019730387 i$ & $-0.000000006094973+0.000000003022158 i$ & $-0.000000000833662+0.000000000458564 i$ \\
$-0.000000209315706-0.000000351842042 i$ & $-0.000000033869024-0.000000052991484 i$ & $-0.000000005431044-0.000000007899402 i$ \\
$0.000000352995514-0.000000206922202 i$ & $0.000000053164861-0.000000033539827 i$ & $0.000000007925411-0.000000005385894 i$ \\
$\bar{\xi}_{13}$ & $\bar{\xi}_{14}$ & $\bar{\xi}_{15}$ \\
\hline$-0.000000000113788+0.000000000069034 i$ & $-0.000000000015496+0.000000000010323 i$ & $-0.000000000002105+0.000000000001535 i$ \\
$-0.000000000863573-0.000000001166826 i$ & $-0.000000000136241-0.000000000170926 i$ & $-0.000000000021338-0.000000000024847 i$ \\
$0.000000001170712-0.000000000857397 i$ & $0.000000000171505-0.000000000135398 i$ & $0.000000000024933-0.000000000021224 i$
\end{tabular}

\section{Numerical approximation $\bar{R}$ and even Fourier coefficients $\overline{\mathcal{Q}}_{k}$ :}

Solution \# 1

$$
\begin{aligned}
& \bar{R}=\left[\begin{array}{rrr}
-1.387511870700525 & 10.413957598738442 & -24.345293393676652 \\
34.433483055001410 & 16.747540413772924 & -64.490466526574409 \\
5.910150213980192 & 10.264994080118939 & -29.026695209740538
\end{array}\right] \\
& \overline{\mathcal{Q}}_{0}=\left[\begin{array}{rrr}
1.164908177280166 & -1.803172340423295 & 3.576351013779620 \\
-3.091997467130629 & 0.061210130102117 & 1.988125340690924 \\
2.718036482444605 & 0.825898407183530 & -3.885210588070526
\end{array}\right]
\end{aligned}
$$

$\overline{\mathcal{Q}}_{2}=$

$-0.866294000482876-1.517174563626462 i$
$0.509788425938065-0.714866339135450 i$ $0.509788425938065-0.714866339135450 i$
$0.402372709747515-0.063751212963967 i$

$0.002921350244971-0.041026859510501 i$ $0.661612810285463-0.362088143572158 i$

$0.661612810285463-0.362088143572158 i$
$-0.220722332917557+0.515436548133675 i$

$0.272254735540097+0.193108273854690 i$
$0.346206488506330+0.232974541129058 i$ $0.346206488506330+0.232974541129058 i$
$-0.415259741322708+0.376758862585817 i$

$0.226805195194691+0.164436218751750 i$ $0.132624286729287+0.362238337115273 i$
$-0.396602815786101+0.163955300090018 i$

$0.139141286867885+0.105392333409777 i$ $0.022491378705591+0.297244131793740 i$ $-0.295670715198465+0.034690679906638 i$

$\left[\begin{array}{r}0.074574329861185+0.061221963277495 i \\ -0.021085752655787+0.197514892292071 i\end{array}\right.$ $-0.191963300440361-0.018713774729379 i$

$0.036973044527057+0.033903826569238 i$ $-0.030689170867607+0.117291019360528 i$
$-0.113726808259459-0.031164706653101 i$

Solution \# 4

$$
\begin{gathered}
\bar{R}=\left[\begin{array}{r}
-10.355025789684301 \\
0.899060859561549 \\
-7.222497813952203
\end{array}\right. \\
\overline{\mathcal{Q}}_{0}=\left[\begin{array}{r}
1.023958718010556 \\
-0.573888622357730 \\
0.593641003024321
\end{array}\right.
\end{gathered}
$$

5.074347263132815 .009663080648024
4.473311681603017

-0.675126514730723
0.071633755972287 0.040742151766272

$\overline{\mathcal{Q}}_{2}=$

$0.138024745785573+0.218099611198252 i$ $0.287829114159326-0.633681030790011$ $0.203769059852901+0.107721913625062$

$\overline{\mathcal{Q}}_{4}=$

$0.284838788861365-0.102407807177850 i$ $0.230422023359519-0.053897172370139 i$ $0.015454270152731+0.317283993327913 i$

$$
\overline{\mathcal{Q}}_{6}=
$$

$0.200555829088783-0.035698340068769 i$ $0.247951861450867+0.116888303230541$

$$
\overline{\mathcal{Q}}_{8}=
$$

$0.127848789361473-0.002657587081552 i$ $0.153471968526916+0.148905166236442 i$

$$
\overline{\mathcal{Q}}_{10}=
$$

$0.073450746075024+0.005413998475731 i$ $0.078758641110580+0.123065445235988$

$$
\overline{\mathcal{Q}}_{12}=
$$

$0.039089264765640+0.005851913643466 i$ $0.034950837585746+0.084663275771703 i$

$$
\overline{\mathcal{Q}}_{14}=
$$
$0.019751905247220+0.004450594969025 i$
$0.012928757340301+0.052434619076431 i$ $+0.301224125958044 i$ $0.489880443422970+1.839100806447159$ $-0.537890180980384+0.144035347276835 i$

$-0.598366228696386+0.039927158582337 i$ $-0.736642472712952+0.218493323289657 i$ $0.382525265190417-0.772796160555542 i$

$-0.597856635403175-0.217185503512216 i$ $0.565050894702496-0.524858613861323 i$ $0.697655874103223-0.593956538793055 i$

$-0.417753912756603-0.208768292791024 i$ $-0.256749954059345-0.636445460226098 i$ $0.667238511937733-0.280824220397952 i$

$-0.245560436837712-0.140410932437067 i$ $-0.071921501008139-0.502287082721128 i$ $0.498006841524345-0.080182881735437 i$

$\left.\begin{array}{r}-0.130148022379516-0.083821935619066 i \\ 0.009763089761531-0.331276984497606 i\end{array}\right]$ $0.323798241079228+0.009713985079557 i$

$-0.064567903024736-0.047344137089248 i$ $0.034466100020053-0.197102241654019 i$ $0.192411484019366+0.036313805087712 i$ 
$\left[\begin{array}{r}-0.047826742741476-0.090749687879213 i \\ 0.265817278059553+0.091999384890591 i\end{array}\right.$ $-0.193372062784211+0.147055239961529 i$

$0.025498974224084+0.025507226852369 i$
$0.019926879914556+0.060459834467346 i$ $0.019926879914556+0.060459834467346 i$
$-0.074073542381906+0.029861690000861 i$

$0.008288011670258+0.004860383652844 i$ $-0.022097257512586+0.002295893083263 i$

$\left[\begin{array}{r}0.001688790296409+0.000787492845468 i \\ -0.000094100471388+0.005833802350177 i\end{array}\right.$ $-0.000094100471388+0.005833802350177 i$
$-0.005671743885677-0.000185380438099 i$

$0.000307654059622+0.000124254364919 i$ $\left[\begin{array}{l}-0.000076757144186+0.001291949037968 i \\ -0.001279612235996-0.000102271912113 i\end{array}\right.$

$0.053293732076546+0.018836962984192 i$
$-0.020444526584427+0.264463962191678 i$ $-0.020444526584427+0.264463962191678 i$
$-0.263658578699069-0.024245634946039 i$

$0.008925530239504+0.002730948596921 i$ $-0.003951084971026+0.051179892292212 i$
$0.252122278258324-0.098223334446528 i$ $0.030279421996656+0.406013396545621 i$

$$
\overline{\mathcal{Q}}_{4}=
$$

$0.069941219836411-0.066317758714468$ $0.165349054957407+0.041485865858316 i$ $-0.165349054957407+0.041485865858316 i$

$$
\overline{\mathcal{Q}}_{6}=
$$

$0.012918990885743-0.015956351350131 i$ $0.050549397456237+0.014972378771104 i$ $-0.015042801858593+0.048889031118857 i$

$\overline{\mathcal{Q}}_{8}=$

$0.002170111882739-0.003127608490179$ $0.012210251990343+0.003899231753956 i$ $-0.004112500955533+0.011989324632692$

$$
\overline{\mathcal{Q}}_{10}=
$$

$0.000347668081985-0.000564475242089 i$ $0.002635543312150+0.000855548446007 i$ $-0.000896750134065+0.002613762734525 i$

$$
\overline{\mathcal{Q}}_{12}=1.0 e-03 *
$$

$0.053627551296946-0.097323410654047$ $0.529941590955565+0.167063705717098$ $-0.173355701294813+0.527451834570075$

$$
\overline{\mathcal{Q}}_{14}=1.0 e-03 *
$$

$0.008007810390487-0.016262173402608$ $-0.030934399104456+0.100900542958549 i$

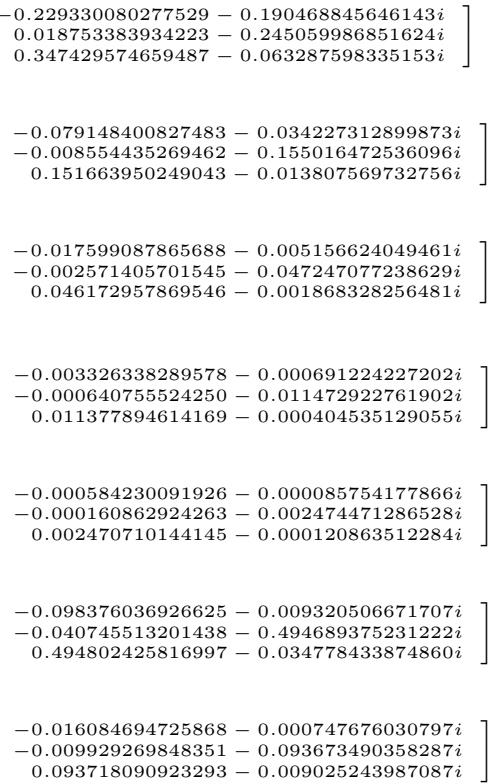

\section{Enclosure of the spectrum and eigenvectors of $R$ :}

Solution \#

\begin{tabular}{r|r|r|r} 
& Stable & & Unstable \\
\hline E.values & -15.109380514113965 & $-1.9711376476 \cdot 10^{-12}$ & 1.442713847447833 \\
& -0.751214982423802 & 0.254365252143146 & 0.262950302967878 \\
E.vectors & -0.336978550953493 & 0.894331168360885 & 0.897737089234849 \\
& -0.567557491695149 & 0.368062603099539 & 0.353447671913741 \\
Rad & $6.5357966401 \cdot 10^{-6}$ & $2.4292063094 \cdot 10^{-4}$ & $2.4457229537 \cdot 10^{-4}$
\end{tabular}

Solution \# 2

\begin{tabular}{r|r|r|r} 
& Stable & & Unstable \\
\hline \multirow{2}{*}{ E.values } & -14.418434214853773 & $-6.3095429563 \cdot 10^{-13}$ & 0.751767548187609 \\
& -0.749706208066872 & 0.206834663675313 & 0.230648743314282 \\
E.vectors & -0.289601764001737 & 0.877249310542877 & 0.889680889496459 \\
& -0.595039007018088 & 0.433189414753372 & 0.394041967399836 \\
Rad & $1.5819989791 \cdot 10^{-8}$ & $2.2544294848 \cdot 10^{-7}$ & $2.2929889758 \cdot 10^{-7}$
\end{tabular}

Solution \# 3

\begin{tabular}{r|r|r|r} 
& Stable & & Unstable \\
\hline E.values & -14.075379469563303 & $-1.9608231416 \cdot 10^{-13}$ & 0.408712802896790 \\
& 0.765749307086543 & 0.150573371977808 & -0.182969022197064 \\
E.vectors & 0.180786590407435 & 0.836967739109956 & -0.859777763658707 \\
& 0.617206778499179 & 0.526129892080275 & -0.476764652668675 \\
Rad & $1.1204301894 \cdot 10^{-9}$ & $1.4128237162 \cdot 10^{-8}$ & $1.4431490181 \cdot 10^{-8}$
\end{tabular}

Solution \# 4

\begin{tabular}{r|r|r|r} 
& Stable & & Unstable \\
\hline E.values & -13.840311255652775 & $-1.4330029932 \cdot 10^{-13}$ & 0.173644588986231 \\
& 0.797501959393221 & 0.078973004231439 & -0.117842783974500 \\
E.vectors & 0.003822364878423 & 0.755631456467173 & -0.792087449202407 \\
& 0.603304246869447 & 0.650218706744094 & -0.598924495309018 \\
Rad & $4.5252927940 \cdot 10^{-10}$ & $6.1203260279 \cdot 10^{-9}$ & $6.2737968625 \cdot 10^{-9}$
\end{tabular}

Solution \# 5

\begin{tabular}{r|r|r|r} 
& \multicolumn{2}{|c|}{ Stable } & Unstable \\
\hline E.values & -13.667080400789002 & $9.0011809244 \cdot 10^{-14}$ & 0.000413734122254 \\
& -0.863856794412098 & 0.232602509848306 & 0.287469286236642 \\
E.vectors & 0.300568560001125 & 0.819824988052658 & 0.854693879140621 \\
& -0.404240002333891 & 0.523242832131249 & 0.432272810190687 \\
Rad & $4.1042307771 \cdot 10^{-9}$ & $1.0966119731 \cdot 10^{-5}$ & $9.9985010904 \cdot 10^{-6}$
\end{tabular}




\section{References}

[1] G. Floquet. Sur les équations différentielles linéaires à coefficients périodiques. Ann. Sci. École Norm. Sup. (2), 12:47-88, 1883.

[2] Carmen Chicone. Ordinary differential equations with applications, volume 34 of Texts in Applied Mathematics. Springer, New York, second edition, 2006.

[3] X. Cabré, E. Fontich, and R. de la Llave. The parameterization method for invariant manifolds. III. Overview and applications. J. Differential Equations, 218(2):444-515, 2005 .

[4] Piotr Zgliczynski. $C^{1}$ Lohner algorithm. Found. Comput. Math., 2(4):429-465, 2002.

[5] Tomasz Kapela and Carles Simó. Computer assisted proofs for nonsymmetric planar choreographies and for stability of the Eight. Nonlinearity, 20(5):1241-1255, 2007.

[6] A. Haro and R. de la Llave. A parameterization method for the computation of invariant tori and their whiskers in quasi-periodic maps: explorations and mechanisms for the breakdown of hyperbolicity. SIAM J. Appl. Dyn. Syst., 6(1):142-207 (electronic), 2007.

[7] À. Haro and R. de la Llave. A parameterization method for the computation of invariant tori and their whiskers in quasi-periodic maps: numerical algorithms. Discrete Contin. Dyn. Syst. Ser. B, 6(6):1261-1300 (electronic), 2006.

[8] A. Haro and R. de la Llave. A parameterization method for the computation of invariant tori and their whiskers in quasi-periodic maps: rigorous results. J. Differential Equations, 228(2):530-579, 2006.

[9] Piotr Zgliczyński and Konstantin Mischaikow. Rigorous numerics for partial differential equations: the Kuramoto-Sivashinsky equation. Found. Comput. Math., 1(3):255-288, 2001.

[10] Nobito Yamamoto. A numerical verification method for solutions of boundary value problems with local uniqueness by Banach's fixed-point theorem. SIAM J. Numer. Anal., 35(5):2004-2013 (electronic), 1998.

[11] Konstantin Mischaikow and Marian Mrozek. Chaos in the Lorenz equations: a computer-assisted proof. Bull. Amer. Math. Soc. (N.S.), 32(1):66-72, 1995.

[12] Warwick Tucker. The Lorenz attractor exists. C. R. Acad. Sci. Paris Sér. I Math., 328(12):1197-1202, 1999.

[13] Hans Koch, Alain Schenkel, and Peter Wittwer. Computer-assisted proofs in analysis and programming in logic: a case study. SIAM Rev., 38(4):565-604, 1996.

[14] Siegfried M. Rump. Verification methods: rigorous results using floating-point arithmetic. Acta Numer., 19:287-449, 2010.

[15] M. T. Nakao. Numerical verification methods for solutions of ordinary and partial differential equations. Numer. Funct. Anal. Optim., 22(3-4):321-356, 2001.

[16] Sarah Day, Jean-Philippe Lessard, and Konstantin Mischaikow. Validated continuation for equilibria of PDEs. SIAM J. Numer. Anal., 45(4):1398-1424 (electronic), 2007. 
[17] Marcio Gameiro and Jean-Philippe Lessard. Rigorous computation of smooth branches of equilibria for the three dimensional Cahn-Hilliard equation. Numer. Math., 117(4):753-778, 2011.

[18] Marcio Gameiro and Jean-Philippe Lessard. Analytic estimates and rigorous continuation for equilibria of higher-dimensional PDEs. J. Differential Equations, 249(9):2237$2268,2010$.

[19] Richard S. Varga. On diagonal dominance arguments for bounding $\left\|A^{-1}\right\|_{\infty}$. Linear Algebra and its Applications, 14(3):211-217, 1976.

[20] R. Krawczyk. Newton-Algorithmen zur Bestimmung von Nullstellen mit Fehlerschranken. Computing (Arch. Elektron. Rechnen), 4:187-201, 1969.

[21] R. E. Moore. A test for existence of solutions to nonlinear systems. SIAM J. Numer. Anal., 14(4):611-615, 1977.

[22] Alexandre Goldsztejn. On the exponentiation of interval matrices. Preprint, 2009.

[23] Edward P. Oppenheimer and Anthony N. Michel. Application of interval analysis techniques to linear systems. II. The interval matrix exponential function. IEEE Trans. Circuits and Systems, 35(10):1230-1242, 1988.

[24] Roberto Castelli and Jean-Philippe Lessard. A method to rigorously enclose eigenpairs of complex interval matrices. Submitted.

[25] S.M. Rump. INTLAB - INTerval LABoratory. In Tibor Csendes, editor, Developments in Reliable Computing, pages 77-104. Kluwer Academic Publishers, Dordrecht, 1999. http://www.ti3.tu-harburg.de/rump/.

[26] Hinke M. Osinga. Nonorientable manifolds in three-dimensional vector fields. Internat. J. Bifur. Chaos Appl. Sci. Engrg., 13(3):553-570, 2003.

[27] A. Arneodo, P. H. Coullet, E. A. Spiegel, and C. Tresser. Asymptotic chaos. Phys. D, 14(3):327-347, 1985.

[28] Mark E. Johnson, Michael S. Jolly, and Ioannis G. Kevrekidis. Two-dimensional invariant manifolds and global bifurcations: some approximation and visualization studies. Numer. Algorithms, 14(1-3):125-140, 1997. Dynamical numerical analysis (Atlanta, GA, 1995).

[29] INTLAB Codes, http://www.math.rutgers.edu/ lessard/floquet 